\title{
ADAM10- and presenilin 1/g-secretase-dependent cleavage of PTPRT mitigates Neurodegeneration of Alzheimer's disease
}

\section{Siling Liu}

Kunming Institute of Zoology Chinese Academy of Sciences

Zhongyu Zhang

Peking University

Lianwei Li

Kunming Institute of Zoology Chinese Academy of Sciences

Li Yao

Kunming Institute of Zoology Chinese Academy of Sciences

\section{Zhanshan Ma}

Kunming Institute of Zoology Chinese Academy of Sciences

Jiali Li ( $\nabla_{\text {jialili@bjmu.edu.cn ) }}$

Peking University https://orcid.org/0000-0002-0039-3671

\section{Research article}

Keywords: ADAM10-, Alzheimer's disease, PTPRT

Posted Date: March 30th, 2021

DOl: https://doi.org/10.21203/rs.3.rs-327896/v1

License: (9) (i) This work is licensed under a Creative Commons Attribution 4.0 International License. Read Full License 


\section{Abstract}

\section{Background}

PTPRT (receptor-type tyrosine-protein phosphatase T), as a brain-specific type 1 transmembrane protein, plays an important function in neurodevelopment and synapse formation. However, whether PTPRTdependent signaling is involved in Alzheimer's disease (AD) remains elusive.

\section{Methods}

We have begun to address these questions by investigation of the relationship between Ab and PTPRTdependent signaling in both postmortem human AD brain tissues, APP/PS1 mice, and different cell lines using biochemical and histological methods. Using RNAseq we analyzed gene expression regulated by PTPRT intracellular domain (PICD). We further assessed the function of PICD in AD brain using neuropathology, electrophysiology and animal behavior testing after conduction on APP/PS1 mice with overexpressing PICD in the hippocampus.

\section{Results}

A significant downregulation in the levels of PtprtmRNA and protein was found in both human AD and disease model mouse brains. PTPRT intracellular domain (PICD), which was released from ADAM10- and presenilin 1-/g-secretase-dependent cleavage of PTPRT, efficiently translocated to the nucleus via a conserved nuclear localization signal. Inhibition of nuclear-localization of PICD via the mutation of its nuclear localization signal (NLS) leads to accumulation of phosphorylated signal transducer and activator of transcription 3 (pSTAT3), which is a substrate of PTPRT and eventually resulted in neuronal cell death. RNA sequencing reveals that expression of the PICD alone can profoundly alter the expression of genes associated with synapse function and dephosphorylation et al. Overexpression of PICD alone not only significantly decreases the level of phosph-STAT3 ${ }^{\mathrm{Y} 705}$ and Ab deposition in the hippocampus of APP/PS1 mice, but also improves synaptic function and behavioral deficits in APP/PS1 mice.

\section{Conclusions}

Altogether, these findings suggest that a novel role of the ADAM 10- and presenilin 1-/g-secretasedependent cleavage of PTPRT in the events can mitigate neurodegeneration of AD and moderate Alzheimer's pathogenesis.

\section{Background}

Extracellular deposition of the amyloid- $\beta$ peptide is a major hallmark pathology for both early-onset and late-onset Alzheimer's disease, and it has been taken as the culprit in the appearance of synaptic dysfunction, neuronal degeneration and loss according to the amyloid hypothesis. The amyloid- $\beta$ peptide $(A \beta)$ is a fragment of a large type I membrane protein known as the amyloid precursor protein (APP). Mechanically, A $\beta$ is released from APP by the combined actions of either the a secretase (a disintegrin 
and metalloproteinase domain-containing protein 10/17, ADAM10/17) or $\beta$ secretase (BACE1) in the juxtamembrane region followed by cleavage with the $y$ secretase/presenilin-1 complex, resulting in secretion of $\mathrm{i}$ ) the soluble N-terminus of APP (SAPP), ii) either P3 or the neurotoxic amyloid- $\beta$ peptide, and iii) a cytoplasmic peptide known as the APP intracellular domain (AICD)[1]. In addition to APP, some other type 1 transmembrane proteins are subject to similar processing, which is also called regulated intramembrane proteolysis (RIP)[2]. RIP, as an evolutionally conserved signaling mechanism, is very important for organisms[2]. The p75 receptor, another important $y$-secretase substrate, is also affected in $A D$ pathogenesis as the released intracellular domain can induce neuronal apoptosis after translocating to the nucleus[3, 4]. TREM2, another type 1 transmembrane protein and AD risk-factor gene, can also be cleaved by $a / \gamma$-secretase action[5]. The ectodomain sTREM 2 released by ADAM10 induces inflammatory responses and enhances microglial survival[6, 7], while the function of the intracellular domain is uncertain. RIP mediated signaling is also important for neurodevelopment, as illustrated by yet another $\mathrm{Y}^{-}$ secretase substrate, Notch. After binding with its ligands, cleavage of Notch liberates its intracellular domain (NICD), which enters the nucleus to regulate developmental gene expression. The Notch pathway has been shown to regulate neurogenesis, synaptic plasticity and long-term memory[8-10]. Sequential cleavage of EphA3 by $a$ - and $y$-secretases mediates axon elongation, and the released intracellular domain of EphA3 is essential for the process[11].

PTPRT, a receptor-type protein tyrosine phosphatase, is a type 1 transmembrane protein with two intracellular catalytic domains. It is predominantly expressed in the brain of mammals. Clinical and sequencing studies have shown that mutations in the Ptprt gene would cause intellectual development and neurological disorders[12]. Several GWAS studies show that PTPRT is heavily involved in neurodevelopmental disorders[12-15]. PTPRT works as a regulator for synaptic formation via its dephosphorylation activity[16]. PTPRT could also regulate dendritic arborization and fusion of synaptic vesicles by dephosphorylating BCR and Syntaxin-binding protein 1 during neurodevelopment[17]. At the molecular level, PTPRT plays a crucial role in synapse formation by interacting with cell adhesion molecules[16]. Dephosphorylation of BCR protein by PTPRT regulates neuronal dendritic arborization[17]. PTPRT is also a potent phosphatase for STAT3 at Y705 residue[18].

STAT3, as a transcriptional factor, responds promptly to environmental stimulation by internal and external changes, which is important for cellular adaption[19, 20]. The rapid coordination between tyrosine kinases and phosphatases is important for the process. After being phosphorylated at $Y 705$ by JAK, pSTAT $3^{\mathrm{Y} 705}$ forms dimers and then accumulates in the nucleus, activating the transcription of a wide range of genes, including some oncogenes. Mutations or epigenetic silencing in the Ptprt gene could promote tumor growth due to the hyperactivation of STAT3[21, 22]. In the central nervous system, STAT3 also plays essential roles. STAT3 modulates neurogenesis and differentiation during brain development[23, 24], and activation of STAT3 is neuroprotective against brain ischemia by upregulating survivin and Mn-SOD[25]. In neurodegenerative diseases, neural STAT3 activation is a common phenomenon, and more evidence has found it detrimental for neuronal survival[26]. Activated STAT3 has 
also been found to facilitate long-term depression (LTD) in the AD mouse model and promote b-amyloid production by upregulating $B A C E 1$ expression in neurons[27].

In this study, we found that the downregulated mRNA and decreased protein levels of PTPRT led to the accumulation of PSTAT3 ${ }^{\mathrm{Y} 705}$ in the brains of human AD and model mice. We further identify that PTPRT, a regulator of STAT3 in the mammalian brain, is a novel substrate for ADMA10 and presenilin 1/gsecretase. After cleavage by ADAM10 and presenilin 1/g-secretase, the intracellular domain of the released PTPRT (PICD) is translocated to the nucleus and regulates gene expression via dephosphorylation of pSTAT $^{\mathrm{Y} 705}$. Overexpression of PICD alone significantly reduces PSTAT3 ${ }^{\mathrm{Y} 705}$ and Ab deposition, and improves synaptic function and behavioral deficits in APP / PS1 mice.

\section{Materials And Methods}

\section{Antibodies and Reagents}

Antibodies against PTPRT (MABS1158 for WB and HPA017336 for $\mathrm{IHC}$ ) and Flag (F3165) were from Sigma; pSTAT3 (Y705) (AP0070), ADAM10 (A10438) and ADAM17 ( A0821) antibodies were from ABclonal; antibodies against STAT3 (ab68153), a-tubulin (ab7291), $\beta$-actin (ab8227), Presenlin-1 (ab15456) and Map2 (ab32454 and ab11267) were obtained from Abcam; antibodies against cleavedCaspase3 (\#9661) , a-tubulin: (\#3873S), histon3 (\#9715) were purchased from Cell Signaling Tech; antibodies against the 6E10 (SIG-393200) antibody were from Covance. The secondary antibodies used for immunocytochemistry were as follows: chicken anti-mouse or rabbit Alexa 488; donkey anti-mouse or rabbit Alexa 594 (Invitrogen, Eugene, OR); all were used at a dilution of 1:500. ProLong ${ }^{\text {TM }}$ Diamond Antifade Mountant with DAPI (Thermo Fisher Scientific, P36962) was used as a nuclear counterstain at 1 $\mu \mathrm{g} / \mathrm{ml}$. DAPT ( $\mathrm{\gamma}$-secretase inhibitor, D5942), Thioflavine S (T1892), Anti-Digoxigenin-AP (Fab fragments, 11093274910), and Phorbol 12-myristate 13-acetate (PMA, p8139) were ordered from Sigma-Aldrich.

\section{Constructs}

pLV-Ptprt-FL-mcherry and pLV-Ptprt-ICD-mcherry were constructed from the backbone of pLV-mcherry (Addgene, \#36084). AAV-hSyn1-nls-luciferase-FLAG (control) and AAV-hSyn1-Ptprt-ICD-FLAG were constructed from the backbone of AAV-hysn1-GCaMP6s-P2A-nls-dTomoto (Addgene gene, \# 51084). AAVhysn1-GCaMP6s-P2A-nls-dTomoto was digested with BamH1 and EconR1. nls-luciferase-FLAG and PTPRT-ICD-FLAG were PCR amplified separately and the ligated with the backbone after BamH1 and EconR1 digestion to make AAV-nls-luciferase-FLAG and AAV-hSyn1-Ptprt-ICD-FLAG. nls and FLAG sequence were added by PCR amplification. pcDNA3.1-Ptprt was cloned from mouse brain RNA by SuperScript III one-step RT-PCR system (Invitrogen). Lentiviral shRNAs against mouse ptprt were constructed in pLKO.1 (Invitrogen) and transfected with pMD2g and psPAX into HEK293T cells for viral package. The sequences of shRNA targets can be found in Supplementary Table 1.

\section{Viral Preparation}


Lentivirus preparation, the construct was co-transfected to HEK293T cells pMD2g and psPAX. The original medium was changed with the fresh one. 48 hours after that, the medium was collected. The lentivirus particles were concentrated by centrifuging at 25,000 rpm for 2 hours. The production of lentiviral particles up to $109 \mathrm{IU} / \mathrm{ml}$ was purified by Ultra-Pure Lentivirus purification kits (Applied Biological Materials Inc. Vancouver). AAV preparation, the constructs were packaged into AAV9 by the MIRCen viral production platform. Briefly, viral particles were produced by transient co-transfection of HEK-293T cells with an adenovirus helper plasmid, an AAV packaging plasmid carrying the rep2 and cap9 genes, and the AAV2 transfer vector containing the above-mentioned expression cassettes by PEI. $72 \mathrm{~h}$ following transfection, virions were purified and concentrated from cell lysate and supernatant by ultracentrifugation on an iodixanol density gradient followed by buffer exchange to PBS, $0.01 \%$ Pluronic via a 100kd Amicon Centrifugal filter unit (Merck-Millipore, Darmstadt, Germany). Concentration of the vector stocks was estimated by quantitative PCR and expressed as viral genomes per $\mathrm{ml}$ of concentrated stocks $(\mathrm{vg} / \mathrm{ml})$.

\section{Human brain tissues}

Human autopsy paraffin-embedded $10 \mu \mathrm{m}$ brain sections were from the following sources with approval from the appropriate local regulatory authorities. We examined 16 case-patients graciously provided by the University of Pittsburgh Alzheimer's Disease Research Center (ADRC) brain bank with approval from the Committee for Oversight of Research and Clinical Training Involving Decedents (CORID). Basic information has been described as our previous study[28]. Additional frozen tissue was a generous gift of the ADRC at Washington University in St. Louis (Grant P50-AG-05681) with approval from the Neuropathology Core (protocol \#T1016) at Hongkong University of Science and Technology.

\section{Animals}

Animal studies were performed with age- and sex-matched wild-type and APP/PS1 mice. APP/PS1 mice carry two different human genes harboring disease-associated mutations: $\mathrm{PS}_{1 \mathrm{~m} 146 \mathrm{v}} / \mathrm{APP}_{\text {swe. }}$. Breeding stock was purchased from The Jackson Laboratory. Animals were housed and bred at the animal facility of the Kunming Institute of Zoology. All animal experiments were conducted under license from the Kunming Institute of Zoology and were approved by the Animal Care and Use Committee of the Kunming Institute of Zoology, Chinese Academy of Sciences (SMKX-SQ-20170628).

\section{Bioinformatic analysis}

For human PTPRT expression analysis, we followed the method from a published paper[29]. Briefly, 20 GSE series of expression data from $684 \mathrm{AD}$ and 562 control human postmortem brain samples was renormalized, and then the expression of PTPRT was extracted and analyzed. For correlation analysis between AD pathological burden and the level of PTPRT expression, a GEO dataset GSE64398 was used[30], and simple linear regression analysis was conducted. To analyze the PTPRT expression in the hippocampus of AD patients at various stages of severity, we used GEO dataset GSE1297[31]. 


\section{Mouse embryonic fibroblasts (MEFs), N2a, and HEK293T cell culture}

All the cells (MEF, N2a and HEK293) were plated onto tissue culture dishes in DMEM (Invitrogen) supplemented with $10 \%$ FBS and $1 \%$ Pen/Strep (Gibco) at $37^{\circ} \mathrm{C}$ and $5 \%$ CO2. PEl $(1 \mathrm{mg} / \mathrm{ml})$ was used for the transfection of HEK293T (WT or mutant) cells. Lentivirus infection of MEF and N2a cells was performed at the second day of subculture when the cell confluence reached $70 \%$. Polybrene (final concentration is $8 \mu \mathrm{g} / \mathrm{mL}$ ) was used to facilitate the infection.

\section{Primary Neuronal Cultures}

Mouse embryonic cortical neurons were isolated by standard procedures. All embryos pups from APP/PS1 $x$ WT mating were harvested and treated separately then retrospectively genotyped by PCR. Isolated E16.5 embryonic hippocampus was treated with 0.25\% Trypsin-EDTA and dissociated into single cells by gentle trituration. Cells were suspended in Neurobasal medium supplemented with B27 and 2 $\mathrm{mM}$ glutamine, then plated on coverslips or dishes coated with poly-L-Lysine $(5 \mathrm{mg} / \mathrm{mL})$. The cells were seeded at $1 \times 10^{4}$ cells $/ \mathrm{cm}^{2}$. All cultures were grown for a minimum of 7 days in vitro (DIV) before any treatment. All viral infections were carried at DIV5. Primary neurons were infected using a multiplicity of infection (moi) between 5 and 10 to provide an efficiency of infection above $70 \%$. All data were collected and analyzed in a double-blind manner.

\section{Immunohistochemistry}

For DAB/bright field staining, all paraffin-embedded human sections were deparaffinized in xylene and then rehydrated through graded ethanol to water. The sections were pretreated with $0.3 \%$ hydrogen peroxide in methanol for $30 \mathrm{~min}$ to remove endogenous peroxidase activity, rinsed in Tris-buffered saline (TBS), and then treated with $0.1 \mathrm{M}$ citrate buffer in a microwave at sufficient power to keep the solution at $100^{\circ} \mathrm{C}$ for $20 \mathrm{~min}$. Sections were cooled in the same buffer at room temperature (RT) for $30 \mathrm{~min}$ and rinsed in TBS. Slides were incubated in 10\% goat serum in PBS blocking solution for $1 \mathrm{~h}$ at RT, after which the primary antibody was applied to the sections that were then incubated at $4^{\circ} \mathrm{C}$ overnight. The sections were washed three times in TBS before applying the secondary antibody for 1 hour at RT. Afterward, sections were rinsed and incubated in Vectastain ABC Elite reagent, and developed using diaminobenzidine according to the protocol of the manufacturer (Vector Laboratories). The sections were counterstained with hematoxylin and mounted in Permount. Control sections were subjected to the identical staining procedure except for the omission of the primary antibody. All sections were analyzed and imaged by using Axiolmager. Z1 (Zeiss), and LSM 780 NLO confocal (Zeiss), microscopes. The collection and analysis of all data were performed in a double-blind manner.

\section{Immunofluorescence}

All paraffin-embedded human sections were deparaffinized in xylene and then rehydrated through graded ethanol to water and PBS. For the mouse, cryostat sections were first rinsed in PBS, Subsequent steps were identical for paraffin and cryostat material. All primary antibodies were diluted in PBS containing 
$0.5 \%$ TritonX-100 and $5 \%$ goat serum and incubated with sections overnight at $4{ }^{\circ} \mathrm{C}$. After rinsing in PBS, they were incubated for 1 hour with the appropriate secondary antibody, which was conjugated with a fluorescent label. All sections were mounted in ProLong ${ }^{\circledR}$ Gold anti-fade reagent with DAPI (Invitrogen) under a glass coverslip. All immunofluorescence images were collected using a Zeiss Olympus IX-81 microscope with either a 20x or 40x objective running Metamorph. Microsoft Excel was used to calculate the fraction of positive clusters. GraphPad Prism was used to perform statistical analysis and to visualize bar charts. Error bars represent S.E.M. All data were collected and analyzed in a double-blind manner.

\section{Protein extraction and western blot}

Brain tissues were lysed using 1× RIPA buffer (Sigma Aldrich), complemented with PIC (Protease Inhibitor Cocktail, Cat \#11836153001, Roche) and Phosphatase Inhibitor Cocktail (Cat \#11836153001, Roche). After protein quantification, samples were boiled for $10 \mathrm{~min}$ in SDS loading buffer (1:1 ratio). An aliquot (up to $50 \mu \mathrm{g}$ ) of the resulting sample was run on an SDS-PAGE gel and then transferred to a HybondPVDF membrane (Amersham Pharmacia). The membrane was blocked in $5 \%$ milk for 1 hour at room temperature and incubated overnight with the appropriate primary antibody re-suspended in $5 \%$ milk in TBST at $4{ }^{\circ} \mathrm{C}$. Following three washes in TBST, the membrane was incubated for 2 hours at room temperature with the appropriate secondary antibody re-suspended in 5\% milk in TBST at room temperature, followed by 4 washes using TBST. Signal ECL (Pierce) amplification was detected by Tanon 5200 Multi chemiluminescent image system (Tanon, Shanghai, China). Signal intensity was quantified with Image J (National Institute of Health).

\section{RNA sequencing}

Libraries were prepared from the RNAs of three 3 biologically independent experiments. Sequencelibraries of each sample were equimolarly pooled and sequenced on an Illumina NextSeq 500 instrument (High Output, 75 bp, Single Reads, v2) at the core facility of the Kunming Institute of Zoology. Differentially expressed genes were uploaded into the IPA software (Ingenuity Systems, http://www.ingenuity.com). An IPA core analysis was performed focusing on both up- and downregulated molecules and setting the log ratio (LR) cutoff $\geq 0.58$ (fold change $\geq 1.5$ ) and the FDR cutoff $\leq 0.0005$ (range 0.0-0.0005). FastQC (v0.119) and Fastx-Toolkit (v0.0.13) were used to clean the sequencing data. We use Tophat (v2.1.1) to align clean sequencing reads to the exon of the reference genome (Mus musculus (house mouse), GRCm38.p6). The unmapped reads were split into shorter fragments and then aligned to splice junctions between exons. Cufflinks (v2.1.1) use the alignment files from Tophat to assemble and reconstruct the transcriptome. The transcript abundances were estimates by Cuffnorm and differential expression of transcripts performed by Cuffdiff.

\section{Data availability}

RNA-seq raw data have been deposited in NCBI's Gene Expression Omnibus and are accessible through GEO Series accession number GSE159943 (https://www.ncbi.nlm.nih.gov/geo/query/acc.cgi? acc=GSE159943). 


\section{Virus production and stereotactic intracranial injection}

Viral preparation and hippocampal microinjection were conducted as previously described[28]. Briefly, the high adenoviral PICD viral particle titers were prepared with the Adenoviral Expression system and purified by Adenoviral Purification Kits (Applied Biological Material Inc. Vancouver). Stereotaxic intrahippocampal infusions were delivered to wild-type and APP/PS1 mice (9 months of age) under isoflurane anesthesia. Mice were positioned in a Kopf stereotaxic apparatus and burr holes drilled into the skull. A 5$\mathrm{ml} \mathrm{Hamilton}$ syringe fitted with a 33-gauge needle was lowered into the hippocampus for viral particles delivery. The coordinates relative to bregma: anteroposterior, $-2.18 \mathrm{~mm}$; mediolateral, $\pm 2.30 \mathrm{~mm}$; and dorsoventral, $-2.10 \mathrm{~mm}$. Each hippocampus was injected with $1 \mu \mathrm{l}$ of the adenovirus-associated viral particles using a Hamilton syringe. The injection rate was $0.2 \mu \mathrm{l} / \mathrm{min}$. All experiment as indicated above for the mice were carried out 3 months after injection. All data were collected and analyzed in a doubleblind manner.

Field potential recording and LTP

Preparation and recording of hippocampal brain slices were described as previously[32]. Briefly, mice of 12-month-old wild type and APP/PS1 one week after completion of behavioral testing are decapitated under isoflurane anesthesia, and the brains removed. Extracellular recordings of field excitatory postsynaptic potentials (fEPSPs) were made with artificial cerebrospinal fluid (ACSF)-filled glass electrodes (5-10 $\mathrm{mm}$ tip diameter). Test stimuli $(0.1 \mathrm{~ms}$ ) were delivered with a bipolar platinum/iridium stimulating electrode at 1 min intervals, except for specialized protocols that elicited changes in synaptic strength (see below). For recordings of CA1 activation by Schaffer collateral stimulation, recording and stimulating electrodes were both placed in stratum radiatum. Each experiment was begun by obtaining input-output relationships to establish the strength of baseline synaptic transmission. A Grass S8800 stimulator connected to a Grass PSIU6 photoelectric stimulus isolation unit was used to deliver a series of increasing-intensity constant-current pulses. Current magnitude was adjusted to elicit responses ranging from just suprathreshold to near maximal. Following this, stimulus intensity was adjusted to evoke fEPSPs $30 \%-40 \%$ of maximum, typically $30-40 \mathrm{~mA}$. To elicit LTP, TBS was used. A single TBS consisted of 12 bursts of four $100 \mathrm{~Hz}$ pulses spaced $200 \mathrm{~ms}$ apart. Response magnitude was quantified

with the slope of the field potential. Statistics Test of significance were performed using either ANOVA, or paired and unpaired t-tests as appropriate.

\section{Morris Water Maze}

WT mice and APP/PS1 mice in the AAV-control and PICD groups were trained in an open circular pool (diameter: $170 \mathrm{~cm}$ ) filled with water at a depth of $30 \mathrm{~cm}$ and maintained at $22^{\circ} \mathrm{C} \pm 1^{\circ} \mathrm{C}$ as described previously[33]. For data collection, the maze was divided into four equal quadrants (I-IV) by designating two orthogonal axes, the end of which demarcated four cardinal points: north, south, east, and west. Mice that failed to find the platform within $60 \mathrm{~s}$ were placed on it for $10 \mathrm{~s}$, the same period as was allowed for the successful animals. White geometric figures, one hung on each wall of the room, were used as 
external visual clues. Behavior was evaluated by direct observation and analysis of videotape-recorded images. Variables of time (escape latency) and quadrant preference and entries were analyzed in all the trials of the tasks. The escape latency was readily measured with a stopwatch by an observer unaware of the animal's genotype and confirmed during the subsequent video-tracking analysis. In the probe trial, the time spent and number of entries in each of the four quadrants were also measured retrospectively by means of the automated video-tracking analysis.

\section{Statistics}

All data were obtained under the same conditions. And all experiments and analysis were performed in a double-blind manner. No randomization was performed to allocate subjects in the study. No sample calculation was performed. No exclusion criteria were pre-determined. No animal was excluded based on the exclusion criteria or died during experiments. Data were presented as the mean \pm S.E.M. $P$ values describing significance were based on Student $t$ tests (2-tailed; $a=0.05$ ) or analysis of repeatedmeasures two-way ANOVA. $P$ values less than 0.05 indicate statistical significance. GraphPad Prism 8.0 was used to perform ANOVAs and $t$-tests and to visualize bar charts.

\section{Results}

\section{The level of PTPRT expression decreased in the brains of human AD and model mice}

PTPRT is highly expressed in the mammalian brain and regulates STAT3 phosphorylation. Its function in neurodevelopment has been reported. To investigate whether and how alteration in PTPRT signaling occurs in age-related neurodegenerative diseases such as AD remains unclear, first, the RNA-seq data set of human AD brain samples were downloaded from NCBI and analyzed. Surprisingly, Ptprt mRNA expression was significantly downregulated in several brain regions of human $A D$ including $P F C, H P, E C$, and TC compared to that of controls (Fig. 1a, Figure S1a). Next, using immunohistochemistry (IHC) the levels of PTPRT in age- and sex-matched postmortem human control and AD brain samples were observed. Indeed, a significant decrease in the intensities of PTPRT signals was found in the PFC and hippocampus of AD compared to age-matched control samples (Fig. 1b-c). Western blot data from human AD brain tissues further confirmed a decrease in the protein level of PTPRT full length and two CTF cleavages (Fig. 1d-e).

Next, to assess whether AD mouse models can recapitulate decreased mRNA expression and protein level of PTPRT found in human AD brain, first, RNA-seq data from 3xTg and APP/PS1 mouse brain samples were downloaded from NCBI, and the dynamics of Ptprt expression in APP/PS1, 3xTg, and wild type mice were analyzed. Interestingly, the levels of Ptprt mRNA showed significantly decrease in 1-year-old 3xTg and APP/PS1 mouse brains. Interestingly, the expression of ptprt would reach a peak at 4-month-old, and then decreases gradually with the age growing in both mouse cortex and hippocampus. While wild type, APP/PS1, and 3xTg mice showed similar patterns, the mRNA expression level of Ptprt in both APP/PS1 and $3 x \mathrm{Tg}$ mice is much lower than that of wild type (Fig. 1f, Figure S1b). We further found that the level 
of Ptprt expression showed a significant negative correlation with $b$-amyloid $\left(r^{2}=0.1796, P=0.0064\right)$ and pathological burden $\left(r^{2}=0.2310, P=0.001\right)$ in APP/PS1 mice (Fig. 1g). Despite a strong age- and A $\beta$ pathology-related decrease in the levels of Ptprt expression, there is no correlation between Ptprt expression and tau pathology in 3xTg (Figure S1c). Next, using immunohistochemistry and Western blot we further verified the decrease in the levels of PTPRT in PFC and CA1 of APP/PS1 mice (Fig. 1h-k).

\section{The level of pSTAT3 ${ }^{\mathrm{Y} 705}$ increased in the brains of human AD and model mice}

Accumulation of pSTAT3 ${ }^{\mathrm{Y} 705}$ has been reported in the brain of neurodegenerative diseases. To detected whether decreased PTPRT could affect the level of pSTAT3 ${ }^{\mathrm{Y} 705}$ in AD brains, first, using immunostaining we examined pSTAT3 ${ }^{\mathrm{Y705}}$ in the brains of human AD and APP/PS1 mice. Indeed, the intensities of pSTAT3 ${ }^{\mathrm{Y} 705}$ were significantly upregulated in PFC and hippocampal CA1 of human AD and APP/PS mouse brains compared to control groups (Fig. 2a-b, 2e-f). Western blot data from human and mouse brain tissues further confirmed the decrease in the level of pSTAT3 ${ }^{\mathrm{Y} 705}$ in AD brains (Fig. 2c-d, 2g-h). Lastly, using primary cortical neurons we observed a downregulated PTPRT and an upregulated pSTAT3 ${ }^{\mathrm{Y} 705}$ after Ab treatment at DIV14 (Fig. 2i-k). In the late stage of AD, the brain is characterized by serious neurodegeneration and neural loss in the hippocampus, which is the most vulnerable brain area to Ab deposition. This data suggests that the downregulation of PTPRT expression could lead to accumulation of PSTAT3 ${ }^{\mathrm{Y} 705}$ in the brains of human $A D$ and model mice.

\section{PTPRT is a novel substrate of ADAM10- and presenilin 1/g-secretase}

Extracellular domain shedding in the juxtamembrane region is necessary for the subsequent cleavage by presenilin 1/g-secretase complex. To determine whether PTPRT could undergo sequential processing, we treated the HEK293 cells overexpressed with C terminal FLAG-tagged PTPRT (intracellular domain) with different compounds. DAPT ( $\mathrm{y}$-secretase inhibitor) could increase the accumulation of the smaller $\mathrm{C}$ terminal region fragment (CTF2) while it didn't affect the bigger $C$ terminal region fragment (CTF1), which is already known to be produced by furin cleavage. PMA, as an a-secretase activator via the PKC pathway, could add to CTF1 accumulation by DAPT inhibition. The treatment with ADAM/matrix metalloproteinase inhibitor GM6001 could decrease the CTF2 accumulation (Fig. 3a-b). Treatment with proteasome inhibitor MG132 solely helped us detect the accumulation of intracellular fragments (PICD), which is a little bit smaller than CTF2 (Red arrow represents PICD). We further examined endogenous PTPRT cleavage in primary neurons with an antibody against the PTPRT C-terminal. As expected, a similar result was found (Fig. 3c-d). ADAM10 and ADAM17 are the two most important a-secretases responsible for the ectodomain shedding. We tested g-secretase cleavage in ADAM10 KO and ADAM17 KO HEK293 cells[34], respectively. As we could see, CTF2 didn't accumulate significantly after DAPT treatment in ADAM10-KO cells compared with WT cells (Fig. 3e-f). It suggested that ADAM10 rather than ADAM17 is the major secretase for PTPRT shedding. For further verification of PTPRT cleavage by $\mathrm{Y}^{-}$ secretase, we transfected wild type (MEF-WT) or Psen $1^{-/} / P$ sen $2^{-1}$ double knockout mouse embryonic fibroblasts (MEF-DKO) with mouse Ptprt construct. PTPRT CTF2 was absent in WT cells while present in 
DKO cells (Fig. 3g-h). As the main component of the $\mathrm{y}$-secretase complex for intramembrane proteolysis, presenilin 1 is found to cut $\gamma$-secretase substrates without the help of presenilin 2. We expressed Psen 1 delivered by lentivirus to see if it could recuse the processing. Indeed, PTPRT CTF2 could be detected in Psen 1 expressed MEF cells. Furthermore, $\gamma$-secretase could cleave PTPRT and generated an intracellular domain (PICD) at $37^{\circ} \mathrm{C}$ in plasma membrane separated from Ptprt-overexpressed HEK293 cells; and the processing could also be inhibited by DAPT in which the PICD signals were diminished in contrast to an increased level of CTF2 (Figure S2a-b). Together, those results demonstrate the sequential cleavage of PRPRT by ADAM10 and presenilin1/Y-secretase.

pSTAT3 ${ }^{\mathrm{Y} 705}$ is dephosphorylated by PTPRT in cancer cells. Nevertheless, Ptprt is preferentially expressed in the central nervous system, thus it is worth investigating whether PTPRT dephosphorylates pSTAT3 ${ }^{\mathrm{Y} 705}$ in the brain, especially in neurons. First, we examined the dynamics of PTPRT in the developmental mouse brain. Indeed, the full length of PTPRT showed an increasing trend with the postnatal age (Fig. 3i-j). On the contrary, the level of pSTAT3 ${ }^{\mathrm{Y} 705}$ presented a decreasing tendency. Unlike full length, PTPRT-CTF2, the product of ADAM10 cleavage, also decreases gradually during the postnatal stage, showing efficient cleavage of PTPRT-CTF2 by g-secretase with maturing of the brain. It seems that a low level of PTPRT may be necessary for maintaining a relatively high level of pSTAT3 ${ }^{\mathrm{Y705}}$, and gsecretase mediated processing may be involved in it. A high level of pSTAT3 ${ }^{\mathrm{Y} 705}$ is essential for neurogenesis and development in the early stage, while minimum pSTAT3 ${ }^{\mathrm{Y} 705}$ is needed for adults. Next, to examine whether PTPRT directly dephosphorylates PSTAT3 ${ }^{\mathrm{Y} 705}$, using shRNAs against Ptprt mRNA we knocked down its expression in wild-type primary cortical neurons. As expected, knockdown of Ptprt significantly led to an increase in the level of pSTAT3 ${ }^{\mathrm{Y} 705}$ (Fig. 3k-m), suggesting an important role of PTPRT in dephosphorylation of PSTAT3 ${ }^{\mathrm{Y} 705}$ in neurons.

\section{Membrane to nuclear translocation of PTPRT intracellular domain dephosphorylates STAT3}

Nuclear translocation of the released intracellular domain by $\mathrm{Y}$-secretase plays an important role in the regulation of intramembrane proteolysis-mediated signaling. To examine whether a nuclear localization signal (NLS) in PTPRT is responsible for the nuclear translocation of its intracellular domain, using several different types of online software we analyzed and identified that there was putative NLS (KRRKLAKKQK) at the $\mathrm{N}$ terminal of PICD with a high score (http://mleg.cse.sc.edu/seqNLS). To test whether the released PICD translocate to the nucleus via the leading of NLS, we cloned PICD ${ }^{\text {wt }}$ and PICD deltaNLS (without NLS) gene to a vector fused with mCherry, and transfected it to HEK293 cells (Fig. 4a). Unlike mCherry with whole-cell dispersion, PICD ${ }^{\text {wt }}$-mCherry is specifically distributed in the nucleus. The deletion of the putative NLS led to little accumulation of PICD in the nucleus. Due to rapid degradation by the proteasome, it's hard to demonstrate the presence of PICD in the nucleus directly. As a result, we employed a Gal4-UAS based transcription activation system to confirm the nuclear localization of PICD (Figure S2c-d). The system showed that activation of a-secretase shedding by treatment of PMA increased the transcriptional signaling by more PICD nuclear translocation, while DAPT treatment decreased the PICD mediated nuclear transactivation. 
The PTPRT intracellular fragment contains two phosphatase domains, so it was very interesting to see whether the released ICD after $\mathrm{Y}$-secretase cleavage still takes the dephosphorylation activity. PSTAT3 ${ }^{\text {Y705 }}$ is the most important substrate of PTPRT, and PTPRT regulates cancer behavior by controlling STAT3 transcriptional activity in cancer cells. On the other hand, STAT3 plays a role in regulating neurodevelopment and neurodegeneration. We transfected N2a cells with PICD, and our data demonstrated that PICD decreased the total level of PSTAT3 ${ }^{\mathrm{Y} 705}$ in cell lysate (Fig. 4b). As phosphorylated STAT3 at Y705 has the potential to form a dimer and translocate to the nucleus and then acts as a transcriptional factor. We separated the nuclear component from the cytoplasm and found that PICD could significantly decrease nuclear PSTAT3 ${ }^{\mathrm{Y} 705}$ (Fig. 4c), indicating that nucleus localized PICD could dephosphorylate the nuclear PSTAT3 ${ }^{\mathrm{Y} 705}$ more efficiently and directly.

\section{PICD regulates gene expression}

Cleavage and nuclear import have been described for several membrane receptors, yet this mechanism has previously not been implicated in PTPRT signaling. We, therefore, investigated the possibility that nuclear translocation of the PICD could alter gene transcription beyond its regulatory role in the dephosphorylation of pSTAT3 ${ }^{\mathrm{Y705}}$. To test this hypothesis, we generated inducible isogenic N2a cell lines stably expressing the mCherry-tagged PICD as well as mCherry control and subsequently performed RNA deep sequencing (RNA-seq). We then determined the global transcriptome changes of cells expressing the PICD versus cells expressing mCherry as control. We compute the sum of expression of all genes located in each chromosome. And the differences in gene expression between the control vector and the ICD group were presented (Fig. 5a). If Delta $>0$, the sum of gene expression is up-regulated, otherwise, the sum of gene expression is down-regulated. The expression of chromosome 15 has the largest downregulated expression between the control vector and the ICD group (-27.7\%). The largest differential expression location of chromosome 15 is NC_000081.6:47539988-47540137, which can not be annotated to a defined gene. This location contributes to the main difference in chromosome 15 (the delta expression of chromosome 15 is $42659.0-58997.5=-16338.5$, the delta expression of NC_000081.6:47539988-47540137 is 16273-32655=-16382). The significant difference between genes was defined with $p$-value $<0.05$ and the absolute value of log2Foldchange more than 1 (Fig. $5 \mathrm{~b}$ ). Red points represent significantly down-regulated genes with log2Foldchange $<-1$, green points represent significantly up-regulated genes with log2Foldchange $>1$. The grey points are genes without significant difference between the control vector and the ICD group. Down-regulated and up-regulated genes by PICD expression were presented in the heatmap (Figure S3a-b). The Ptprt gene is significantly up-regulated in PICD group. There are 128 genes with a significant difference between the control vector and PICD, which contains 62 down-regulated and 66 up-regulated genes.

Since the Ptprt gene is located on chromosome 2. We selected the biological process and molecular functions that contain the Ptprt gene (Fig. 5c, Figure S3c). All genes on chromosome 2 to the GO database and KEGG database were then annotated (Fig. 5d, Figure S3d). Indeed, most of those biological processes and molecular functions are related to dephosphorylation, phosphatase, or cell adhesion. The 
annotation of the Ptptrgene in the KEGG database is "receptor-type tyrosine-protein phosphatase T". There are no genes that have the same KEGG annotation as the Ptprt gene. We then annotated all genes on 21 chromosomes $(19+\mathrm{XY})$ to $\mathrm{GO}$ and KEGG databases. There are no genes that have the same KEGG annotation as the Ptprt gene too. But 978 genes have the same GO annotations with the Ptprt gene. Amongst, there is only one gene that has significantly differential expression between the control vector and the ICD group: SMAGP on chromosome 15. The Smagp gene is located on chromosome 15, which has the largest differential expression. We selected the biological process and molecular functions that contain Smagp gene (Fig. 5e, Figure S3e). All genes on chromosome 15 to the $\mathrm{GO}$ database and KEGG database were annotated (Fig. 5f, Figure S3f). Indeed, these biological processes and molecular functions are related to cell adhesion and recognition.

\section{PICD prevents $A \beta$ deposition and pathology in the hippocampus of APP/PS1 mice}

AAV delivery of the Ptprt gene to the brain could be an ideal way to prevent these molecular events in vivo. However, the Ptprt-coding sequence is about $4.4 \mathrm{~kb}$, together with the promoter, ITR, and other regulatory sequences the whole size will exceed the maximum accommodation. PICD with half of the full size makes it a good fit for AAV-packaging. Using microinjection of viral particles containing PICD into the hippocampus of 9-month-old mice we observed its effect on AD pathologies in vivo. The efficiency of PICD viral infection was firstly verified by immunohistochemistry and Western blot analysis. Notably, PICD significantly decreased pSTAT3 ${ }^{\mathrm{Y} 705}$ levels in the hippocampus of APP/PS1 mice (Fig. 6a-c).

Next, to assess whether increased pSTAT3 ${ }^{\mathrm{Y} 705}$ induced by loss of the intracellular domain of PTPRT contributes to Alzheimer's pathogenesis as well as neurodegeneration of $A D$, we decided to examine the effect of the intracellular domain of PTPRT on A deposition in vitro and in vivo. First, primary cortical neurons from wild type and APP/PS1 mouse embryos (E16.5) were infected with lentiviral PICD at DIV 5. Neurons were collected at DIV 14, and immunostained with PSTAT3 ${ }^{\mathrm{Y} 705}$ and $6 \mathrm{E} 10$ antibodies. Indeed, PICD-expressing neurons showed a significant decrease in pSTAT3 ${ }^{\mathrm{Y} 705}$ and $6 \mathrm{E} 10$ signals (Fig. $6 \mathrm{~d}-\mathrm{e}$ ). Next, using the histological and Western blot we examined the effect of overexpression of PICD on Ab deposition and pathology. As expect, the induction of PICD led to a significantly reduced $6 \mathrm{E} 10$ area and decreased cleaved caspase 3 in the hippocampus of APP/PS1 mice compared to controls of the viral vector (Fig. 6b-c, 6f-g).

\section{PICD prevents synaptic dysfunction and behavioral deficits in APP/PS1 mice}

To investigate whether loss of PICD affects the basal synaptic transmission, we generated input/output $(1 / 0)$ curves by measuring field-excitatory by stimulation of the Schaffer collaterals at increasing stimulus intensities at 12-month-old mice. APP/PS1 mice with microinjection of PICD viral-like particles into the hippocampus exhibited bigger fEPSP slopes and amplitudes at all stimulus intensities tested and had significantly increased maximum fEPSPs relative to wild type mice (Fig. 7a). The deficits of long-term potentiation (LTP) in AD mice have been well documented before[35, 36]. We, therefore, investigated LTP in12-month-old wild type and APP/PS1 mice. Due to impaired LTP in the APP/PS1 mice, and the smaller 
absolute fEPSP may account for the LTP deficits. Next, we increased the stimulus intensity of the APP/PS1 mice to match baseline fEPSP magnitudes to those of wild type mice. The stimulus intensity used to elicit LTP in the wild type sections was approximately $30 \%$ of the maximum fEPSP slopes, equating to a value of $₫ 0.45 \mathrm{mV} / \mathrm{ms}$, which is below the max value of the wild type mice. LTP magnitudes in these experiments did not significantly differ in the percentage of potentiation when these stronger stimulus intensities were used, indicating that reduced basal transmission does not likely account for the deficits in LTP in the wild type mice. As with the APP/PS1 mice, raising baseline fEPSPs to wild type levels did not result in significantly different LTP, and thus, the data were pooled. Meanwhile, we also lowered the baseline fEPSP of the wild type mice to match APP/PS1 mice and found no difference in potentiation as compared with the normal LTP protocol. All the data were pooled from the APP/PS1 experiments, where baseline fEPSPs were matched to wild type levels, with the experiments where the normal LTP protocol was used. We also pooled the data from the wild type experiments, where baseline fEPSPs were lowered to APP/PS1 levels, with data from the normal LTP protocol. In consistent with reports from other studies, our pool data showed that the reduced LTP in the APP/PS1 as compared to wild-type mice. Indeed, LTP in the APP/PS1 mice with injected PICD viral-like particles was significantly improved despite also having weaker fEPSPs relative to wild type controls (Fig. 7b).

Next, the learning and spatial memory was further tested using the Morris water maze. The test was conducted in wild type and APP/PS1 mice after one month of microinjection of AAV-PICD into the hippocampus at the age of 9 months. Indeed, In contrast to wild type mice, the total time of exploration in the target quadrant shows that the swimming time of APP/PS1 mice in the target quadrant is

significantly reduced $(P=0.0366, \mathrm{n}=6$ mice). Nevertheless, $\mathrm{PICD}$ is overexpressed group can increase the swimming time of APP/PS1 mice in the target quadrant $(P=0.0069, \mathrm{n}=6$ mice) (Fig. 7c-d). Meanwhile, while the latency of APP/PS1 mice crossing the platform in AAV-control group was significantly increased $(P=0.0367, \mathrm{n}=6$ mice), the latency of mice crossing the platform in the overexpression PICD group was significantly reduced ( $P=0.0236, \mathrm{n}=6$ mice) (Fig. 7e-f). This date suggests that the induction of PICD in the hippocampus of APP/PS1 mice can significantly restore the learning ability and spatial learning memory compared to the control groups.

\section{Discussion}

g-secretase mediated RIP plays an essential role in several biological processes[2]. In this study, as shown in Fig. 8, the type 1 transmembrane protein PTPRT was identified as a novel substrate of ADAM10 and presenilin $1 / \mathrm{g}$-secretase, and preferentially restricted to the brain and lessened neurodegeneration of AD. PTPRT was cleaved by presenilin $1 / \gamma$-secretase after the ADAM10 mediated shedding, released the functional domain PICD. The nuclear translocation of PICD efficiently dephosphorylates PSTAT3 in the nucleus. Moreover, the decreased PTPRT found in the brains of both human AD and APP/PS1 mouse brains led to an excessive nuclear accumulation of pSTAT3. PICD alone not only decreased the pSTAT3 level and Ab deposition, but also could improve behavioral deficits in APP/PS1 mice. 
STAT3 has been identified as a substrate of PTPRT. By dephosphorylating STAT3 at Y705, PTPRT blocks dimerization and nuclear translocation of STAT3 and subsequently inhibits tumorigenesis[22]. AS a result, PTPRT has become a potential therapeutic target for certain cancers. As a central nervous systemspecific protein, PTPRT may have a similar function in the adult brain. Our findings show that PTPRT could wipe phosphate at STAT3 in neurons and it may be a key factor in regulating phosphorylation of STAT3 during brain development. A high level of PSTAT3 is necessary for motor neuron differentiation during the embryonic stage when the Ptprt shows a lower expression level in our result. In the adult mouse brain, high expression of Ptprt matches lower pSTAT3, which indicates the pivotal role of PTPRT in downregulating STAT3 activation.

It has been hypothesized that rapid activation of STAT3 following acute injuries is neuroprotective due to the subsequent transcriptional upregulation of neurotrophic genes[37-39]. However, chronic activation of STAT3 is detrimental, especially in neurodegenerative diseases. Microglial JAK/STAT3 pathway activation was found in the brain of Parkinson's disease, which would initiate the inflammatory responses in the brain and lead to neurodegeneration and neural death ultimately[40, 41]. STAT3 activation triggers astrogliosis in Alzheimer's disease[42], and the deletion of STAT3 in astrocyte would reduce A $\beta$ plaque burden and improve the cognitive performance in APP/PS1 mouse model[43]. A $\beta$ induced accumulation of pSTAT3 in neurons directly activated the apoptotic pathway. The A - -PSTAT3-A $\beta$ cycle may play a pivotal role in the progressive process. Ser727 at STAT3 is another important phosphorylation site which also involved in the STAT3 activation. Previous work showed that the phosphorylation STAT3 (Ser727) would induce the dimer formation, which subsequently led to the phosphorylation of STAT3 (Y705). The nuclear translocation of dimerized PSTAT3 ${ }^{\mathrm{Y} 705}$ upregulated the expression of BACE1 and then promoted the generation of amyloid beta[44]. The inhibition of STAT3 activity may be helpful to reduce the production of b-amyloid and slow the progression. Therefore, inhibitions of the JAK/STAT3 pathway become potential therapeutics for neurodegenerative diseases. However, the risk of adverse effects by nonselective inhibition of STAT3 activity in the whole body still deserved serious consideration. Failure to cross the blood-brain barrier (BBB) is also an important issue for many molecules[45].

Pathological alterations in postsynaptic density composition, glutamatergic synaptic transmission, and dendritic spine are complicated in the process of Alzheimer's disease[46]. In the late stage, the significant loss of neurons exacerbates the situation. Caspase 3 is not only a final executor of apoptotic cell death but also a participator in synaptic dysfunction via a non-apoptotic dependent manner[47-49]. Increased caspase 3 activity in spines mediates GluR1 removal from postsynaptic sites in the hippocampal synapses of Tg2576 mice[50]. In this work, microinjection of AVV-PICD into the hippocampus of APP/PS1 mice significantly reduced the levels of cleaved caspase 3 , though the number of hippocampus neurons was not changed. Overexpression of PICD not only restores the LTP impairment but also improves working memory in APP/PS1 mice. In the future, it will be worth investigating whether the nuclear PICD can directly bind to genomic DNA and sever as a transcriptional regulator directly.

The pathogenesis of Alzheimer's disease is complex, and our understanding of the mechanism still needs to be improved. In the past two decades, therapeutic effects against AD mainly focused on $\beta$-amyloid- 
producing g-secretase, and some of the potent g-secretase inhibitors (GSIs) were found. However, up to now none of these potential drugs were successful in clinical trials. One of the most important reasons is that those GSIs would non-selectively block the cleavage of many other substrates[51], some of which may have essential physiological roles[52]. Once blocked, serious adverse effects would happen, like squamous cell carcinoma[53]. In this study, we found that abnormal ADAM10- and presenilin1/gsecretase-mediated proteolysis of PTPRT is involved in the pathological accumulation of PSTAT3 in the nucleus of neurons in AD brain, and demonstrated that PICD significantly improves neuropathological changes and behavioral deficits in APP/PS1 mice, indicating a potentially novel therapeutic strategy for AD.

\section{Declarations}

\section{Acknowledgments}

We are grateful to Bart De Strooper from the KU Leuven in Belgium for granting Ps1/Ps2 double knockout MEF cells, and Yufang Zheng from Fudan University for granting ADAM10/- and ADAM17-/- HEK293 cells. We also thank Prof. Juming Zhou (Kunming Institute of Zoology) for providing us the constructs for GAL4/UAS System. The authors also thank Dr. Karl Herrup for critical reading the manuscript and constructive suggestion.

\section{Funding}

This work was financially supported by the National Science Foundation of China (91649119 and 92049105) to J.L.; and the Peking University (BMU2019YJ001) to J.L.; and the key basic project of Yunnan Province (E039030401) to J.L.

\section{Author information}

Siling Liu, Zhongyu Zhang, Lianwei Li contributed equally to this study.

\section{Affiliations}

Key Laboratory of Animal Models and Human Disease Mechanisms of Chinese Academy of Sciences \& Yunnan Province, Kunming Institute of Zoology, Chinese Academy of Sciences, Kunming, Yunnan 650223, China

Silin Liu, Li Yao and Jiali Li

National Institute on Drug Dependence, Peking University, Beijing 100191, China

Zhongyu Zhang and Jiali Li

PKU/McGovern Institute for Brain Research, Peking University, Beijing 100191, China 
Jiali Li

Kunming College of Life Science, University of Chinese Academy of Sciences, Kunming, Yunnan 650223, China

Siling Liu and Li Yao

National Resource Center for Non-Human Primates, Kunming Primate Research Center, and National Research Facility for Phenotypic and Genetic Analysis of Model Animals (Primate Facility), Kunming Institute of Zoology, Chinese Academy of Sciences, Kunming, Yunnan 650107, China

Jiali Li

State Key Laboratory of Genetic Resources and Evolution, Kunming Institute of Zoology, Chinese Academy of Sciences, Kunming, Yunnan 650223, China

Lianwei Li, Zhanshan Ma

\section{Contributions}

S.L. and J.L. conceived and designed all experiments. S.L., Z.Z., and L.Y. conducted the majority of experiments including Immunohistochemical analysis, western blot, confocal microscopy, fluorescence assay, electrophysiological and animal assays. Z.Z. contributed in the brain slices recordings. L.L., Z.M., and J.L. performed RNA-seq and data analysis; S.L., Z.Z., and L.Y. performed primary cultures, viral particle preparation and infection, and viral microinjection; S.L., and J. L. drafted the manuscript with input from all other authors. All authors read and approved the final manuscript.

\section{Corresponding author}

Correspondence to Jiali Li

\section{Declarations}

\section{Ethics approval and consent to participate}

We examined 16 case patients graciously provided by the University of Pittsburgh Alzheimer's Disease Research Center (ADRC) brain bank with approval from the Committee for Oversight of Research and Clinical Training Involving Decedents (CORID). All animal experiments were conducted with either license from Kunming Institute of Zoology, and were approved by the Animal Care and Use Committee of Kunming Institute of Zoology, Chinese Academy of Sciences, or the Health Science Center of PKU, and were approved by the Animal Ethics Committee of PKU.

\section{Consent for publication}

Not applicable. 
Competing Interests

The authors declare that they have no competing interests.

\section{Abbreviations}

PTPRT: receptor-type protein tyrosine phosphatase

AD: Alzheimer's disease

Ab: Amyloid-b

APP: Amyloid precursor protein

BACE1: $\beta$ secretase

ADAM10: A Disintegrin and metalloproteinase domain-containing protein 10

ADAM17: A Disintegrin and metalloproteinase domain-containing protein 17

CTF: C terminal region fragment

PICD: PTPRT intracellular domain

DAPT: $y$-secretaseinhibitor

PMA: a-secretase activator

STAT3: signal transducer and activator of transcription 3

NLS: nuclear localization signal

LTP: Long-term potentiation

S.E.M.: Standard error of the mean

\section{References}

1. Thinakaran G, Koo EH: Amyloid precursor protein trafficking, processing, and function. The Journal of biological chemistry 2008, 283(44):29615-29619.

2. Brown MS, Ye J, Rawson RB, Goldstein JL: Regulated intramembrane proteolysis: a control mechanism conserved from bacteria to humans. Cell 2000, 100(4):391-398.

3. Kenchappa RS, Zampieri N, Chao MV, Barker PA, Teng HK, Hempstead BL, Carter BD: Liganddependent cleavage of the P75 neurotrophin receptor is necessary for NRIF nuclear translocation and apoptosis in sympathetic neurons. Neuron 2006, 50(2):219-232. 
4. Yao XQ, Jiao SS, Saadipour K, Zeng F, Wang QH, Zhu C, Shen LL, Zeng GH, Liang CR, Wang J et al: p75NTR ectodomain is a physiological neuroprotective molecule against amyloid-beta toxicity in the brain of Alzheimer's disease. Molecular psychiatry 2015, 20(11):1301-1310.

5. Wunderlich P, Glebov K, Kemmerling N, Tien NT, Neumann H, Walter J: Sequential proteolytic processing of the triggering receptor expressed on myeloid cells-2 (TREM2) protein by ectodomain shedding and $y$-secretase-dependent intramembranous cleavage. The Journal of biological chemistry 2013, 288(46):33027-33036.

6. Zhong L, Xu Y, Zhuo R, Wang T, Wang K, Huang R, Wang D, Gao Y, Zhu Y, Sheng X et al: Soluble TREM2 ameliorates pathological phenotypes by modulating microglial functions in an Alzheimer's disease model. Nature communications 2019, 10(1):1365.

7. Ewers M, Franzmeier N, Suárez-Calvet M, Morenas-Rodriguez E, Caballero MAA, Kleinberger G, Piccio $L$, Cruchaga $C$, Deming Y, Dichgans $M$ et al: Increased soluble TREM2 in cerebrospinal fluid is associated with reduced cognitive and clinical decline in Alzheimer's disease. Science translational medicine 2019, 11(507).

8. Petruccelli E, Feyder M, Ledru N, Jaques Y, Anderson E, Kaun KR: Alcohol Activates Scabrous-Notch to Influence Associated Memories. Neuron 2018, 100(5):1209-1223 e1204.

9. Morrison SJ, Perez SE, Qiao Z, Verdi JM, Hicks C, Weinmaster G, Anderson DJ: Transient Notch activation initiates an irreversible switch from neurogenesis to gliogenesis by neural crest stem cells. Cell 2000, 101(5):499-510.

10. Yeo SY, Chitnis AB: Jagged-mediated Notch signaling maintains proliferating neural progenitors and regulates cell diversity in the ventral spinal cord. Proceedings of the National Academy of Sciences of the United States of America 2007, 104(14):5913-5918.

11. Javier-Torrent M, Marco S, Rocandio D, Pons-Vizcarra M, Janes PW, Lackmann M, Egea J, Saura CA: Presenilin/gamma-secretase-dependent EphA3 processing mediates axon elongation through nonmuscle myosin IIA. Elife 2019, 8.

12. Joanna Bernaciak BW-K, Jennifer Castañeda, Anna Kutkowska-Kaźmierczak, Beata Nowakowska: A novel de novo 20q13.11q13.12 microdeletion in a boy with neurodevelopmental disorders - case report. Dev Period Med 2017, 21(2):91-94.

13. Lim SH, Shin S, Kim MH, Kim EC, Lee DY, Moon J, Park HY, Ryu YK, Kang YM, Kang YJ et al: Depression-like behaviors induced by defective PTPRT activity through dysregulated synaptic functions and neurogenesis. Journal of cell science 2020.

14. Schuurs-Hoeijmakers JH, Vulto-van Silfhout AT, Vissers LE, van de V, II, van Bon BW, de Ligt J, Gilissen C, Hehir-Kwa JY, Neveling K, del Rosario M et al: Identification of pathogenic gene variants in small families with intellectually disabled siblings by exome sequencing. Journal of medical genetics 2013, 50(12):802-811.

15. Thirtamara Rajamani K, O'Neill B, Han DD, Frostholm A, Rotter A, Gu HH: Inactivation of the catalytic phosphatase domain of PTPRT/RPTPp increases social interaction in mice. Autism research :

official journal of the International Society for Autism Research 2015, 8(1):19-28. 
16. Lim SH, Kwon SK, Lee MK, Moon J, Jeong DG, Park E, Kim SJ, Park BC, Lee SC, Ryu SE et al: Synapse formation regulated by protein tyrosine phosphatase receptor $\mathrm{T}$ through interaction with cell adhesion molecules and Fyn. EMBO J 2009, 28(22):3564-3578.

17. Park AR, Oh D, Lim SH, Choi J, Moon J, Yu DY, Park SG, Heisterkamp N, Kim E, Myung PK et al: Regulation of dendritic arborization by BCR Rac1 GTPase-activating protein, a substrate of PTPRT. Journal of cell science 2012, 125(Pt 19):4518-4531.

18. Zhang X, Guo A, Yu J, Possemato A, Chen Y, Zheng W, Polakiewicz RD, Kinzler KW, Vogelstein B, Velculescu VE et al: Identification of STAT3 as a substrate of receptor protein tyrosine phosphatase T. Proceedings of the National Academy of Sciences of the United States of America 2007, 104(10):4060-4064.

19. Peterson WM, Wang Q, Tzekova R, Wiegand SJ: Ciliary neurotrophic factor and stress stimuli activate the Jak-STAT pathway in retinal neurons and glia. The Journal of neuroscience : the official journal of the Society for Neuroscience 2000, 20(11):4081-4090.

20. Nicolas CS, Amici M, Bortolotto ZA, Doherty A, Csaba Z, Fafouri A, Dournaud P, Gressens P, Collingridge GL, Peineau S: The role of JAK-STAT signaling within the CNS. Jak-stat 2013, 2(1):e22925.

21. Lui VW, Peyser ND, Ng PK, Hritz J, Zeng Y, Lu Y, Li H, Wang L, Gilbert BR, General IJ et al: Frequent mutation of receptor protein tyrosine phosphatases provides a mechanism for STAT3 hyperactivation in head and neck cancer. Proceedings of the National Academy of Sciences of the United States of America 2014, 111(3):1114-1119.

22. Peyser ND, Freilino M, Wang L, Zeng Y, Li H, Johnson DE, Grandis JR: Frequent promoter hypermethylation of PTPRT increases STAT3 activation and sensitivity to STAT3 inhibition in head and neck cancer. Oncogene 2016, 35(9):1163-1169.

23. Hong S, Song MR: Signal transducer and activator of transcription-3 maintains the stemness of radial glia at mid-neurogenesis. The Journal of neuroscience : the official journal of the Society for Neuroscience 2015, 35(3):1011-1023.

24. Lee S, Shen R, Cho HH, Kwon RJ, Seo SY, Lee JW, Lee SK: STAT3 promotes motor neuron differentiation by collaborating with motor neuron-specific LIM complex. Proceedings of the National Academy of Sciences of the United States of America 2013, 110(28):11445-11450.

25. Sehara Y, Sawicka K, Hwang JY, Latuszek-Barrantes A, Etgen AM, Zukin RS: Survivin Is a transcriptional target of STAT3 critical to estradiol neuroprotection in global ischemia. The Journal of neuroscience : the official journal of the Society for Neuroscience 2013, 33(30):12364-12374.

26. Wan J, Fu AK, Ip FC, Ng HK, Hugon J, Page G, Wang JH, Lai KO, Wu Z, Ip NY: Tyk2/STAT3 signaling mediates beta-amyloid-induced neuronal cell death: implications in Alzheimer's disease. The Journal of neuroscience : the official journal of the Society for Neuroscience 2010, 30(20):6873-6881.

27. McGregor G, Irving AJ, Harvey J: Canonical JAK-STAT signaling is pivotal for long-term depression at adult hippocampal temporoammonic-CA1 synapses. FASEB J 2017, 31(8):3449-3466. 
28. Zhang Y, Zhang Z, Li L, Xu K, Ma Z, Chow HM, Herrup K, Li J: Selective loss of 5hmC promotes neurodegeneration in the mouse model of Alzheimer's disease. FASEB J 2020, 34(12):16364-16382.

29. Xu M, Zhang DF, Luo R, Wu Y, Zhou H, Kong LL, Bi R, Yao YG: A systematic integrated analysis of brain expression profiles reveals YAP1 and other prioritized hub genes as important upstream regulators in Alzheimer's disease. Alzheimer's \& dementia : the journal of the Alzheimer's Association 2018, 14(2):215-229.

30. Matarin M, Salih DA, Yasvoina M, Cummings DM, Guelfi S, Liu W, Nahaboo Solim MA, Moens TG, Paublete RM, Ali SS et al: A genome-wide gene-xpression analysis and database in transgenic mice during development of amyloid or tau pathology. Cell Rep 2015, 10(4):633-644.

31. Eric M Blalock JWG, Kuey Chu Chen, Nada M Porter, William R Markesbery, Philip W Landfield: Incipient Alzheimer's disease: Microarray correlation analyses reveal major transcriptional and tumor suppressor responses.pdf $>$. Proceedings of the National Academy of Sciences of the United States of America 2004 Feb 17, 101(7):2173-2178.

32. Li J, Han YR, Plummer MR, Herrup K: Cytoplasmic ATM in neurons modulates synaptic function. Curr Biol 2009, 19(24):2091-2096.

33. Vorhees CV, Williams MT: Morris water maze: procedures for assessing spatial and related forms of learning and memory. Nat Protoc 2006, 1(2):848-858.

34. Li J, Liu B, Gao X, Ma Z, CaoSong T, Mei YA, Zheng Y: Overexpression of sigma-1 receptor inhibits ADAM10 and ADAM17 mediated shedding in vitro. Protein Cel/ 2012, 3(2):153-159.

35. Oddo S, Caccamo A, Shepherd JD, Murphy MP, Golde TE, Kayed R, Metherate R, Mattson MP, Akbari Y, LaFerla FM: Triple-transgenic model of Alzheimer's disease with plaques and tangles: intracellular Abeta and synaptic dysfunction. Neuron 2003, 39(3):409-421.

36. Yin X, Takei Y, Kido MA, Hirokawa N: Molecular motor KIF17 is fundamental for memory and learning via differential support of synaptic NR2A/2B levels. Neuron 2011, 70(2):310-325.

37. Dziennis S, Alkayed NJ: Role of signal transducer and activator of transcription $\mathbf{3}$ in neuronal survival and regeneration. Reviews in the neurosciences 2008, 19(4-5):341-361.

38. Wang XL, Qiao CM, Liu JO, Li CY: Inhibition of the SOCS1-JAK2-STAT3 Signaling Pathway Confers Neuroprotection in Rats with Ischemic Stroke. Cellular physiology and biochemistry : international journal of experimental cellular physiology, biochemistry, and pharmacology 2017, 44(1):85-98.

39. Leibinger M, Andreadaki A, Diekmann H, Fischer D: Neuronal STAT3 activation is essential for CNTFand inflammatory stimulation-induced CNS axon regeneration. Cell death \& disease 2013, 4(9):e805.

40. Qin H, Buckley JA, Li X, Liu Y, Fox TH, 3rd, Meares GP, Yu H, Yan Z, Harms AS, Li Y et al: Inhibition of the JAK/STAT Pathway Protects Against alpha-Synuclein-Induced Neuroinflammation and Dopaminergic Neurodegeneration. The Journal of neuroscience : the official journal of the Society for Neuroscience 2016, 36(18):5144-5159.

41. Tian DS, Peng J, Murugan M, Feng LJ, Liu JL, Eyo UB, Zhou LJ, Mogilevsky R, Wang W, Wu LJ: Chemokine CCL2-CCR2 Signaling Induces Neuronal Cell Death via STAT3 Activation and IL-1beta 
Production after Status Epilepticus. The Journal of neuroscience : the official journal of the Society for Neuroscience 2017, 37(33):7878-7892.

42. Ben Haim L, Ceyzeriat K, Carrillo-de Sauvage MA, Aubry F, Auregan G, Guillermier M, Ruiz M, Petit F, Houitte $D$, Faivre $E$ et al: The JAK/STAT3 pathway is a common inducer of astrocyte reactivity in Alzheimer's and Huntington's diseases. The Journal of neuroscience : the official journal of the Society for Neuroscience 2015, 35(6):2817-2829.

43. Reichenbach N, Delekate A, Plescher M, Schmitt F, Krauss S, Blank N, Halle A, Petzold GC: Inhibition of Stat3-mediated astrogliosis ameliorates pathology in an Alzheimer's disease model. EMBO Mol Med 2019, 11(2).

44. Wen Y, Yu WH, Maloney B, Bailey J, Ma J, Marie I, Maurin T, Wang L, Figueroa H, Herman M et al: Transcriptional regulation of beta-secretase by $\mathrm{p} 25 / \mathrm{cdk} 5$ leads to enhanced amyloidogenic processing. Neuron 2008, 57(5):680-690.

45. Butini S, Brogi S, Novellino E, Campiani G, Ghosh AK, Brindisi M, Gemma S: The structural evolution of $\beta$-secretase inhibitors: a focus on the development of small-molecule inhibitors. Current topics in medicinal chemistry 2013, 13(15):1787-1807.

46. Sheng M, Sabatini BL, Südhof TC: Synapses and Alzheimer's disease. Cold Spring Harbor perspectives in biology 2012, 4(5).

47. Porter AG, Jänicke RU: Emerging roles of caspase-3 in apoptosis. Cell death and differentiation 1999, 6(2):99-104.

48. Tanabe K, Nakanishi H, Maeda H, Nishioku T, Hashimoto K, Liou SY, Akamine A, Yamamoto K: A predominant apoptotic death pathway of neuronal PC12 cells induced by activated microglia is displaced by a non-apoptotic death pathway following blockage of caspase-3-dependent cascade. The Journal of biological chemistry 1999, 274(22):15725-15731.

49. Nakajima YI, Kuranaga E: Caspase-dependent non-apoptotic processes in development. Cell death and differentiation 2017, 24(8):1422-1430.

50. Hyman BT: Caspase activation without apoptosis: insight into Abeta initiation of neurodegeneration. Nature neuroscience 2011, 14(1):5-6.

51. Prox J, Rittger A, Saftig P: Physiological functions of the amyloid precursor protein secretases ADAM10, BACE1, and presenilin. Experimental brain research 2012, 217(3-4):331-341.

52. Doody RS, Raman R, Farlow M, Iwatsubo T, Vellas B, Joffe S, Kieburtz K, He F, Sun X, Thomas RG et al: A phase 3 trial of semagacestat for treatment of Alzheimer's disease. The New England journal of medicine 2013, 369(4):341-350.

53. Henley DB, Sundell KL, Sethuraman G, Dowsett SA, May PC: Safety profile of semagacestat, a gamma-secretase inhibitor: IDENTITY trial findings. Current medical research and opinion 2014, 30(10):2021-2032.

\section{Figures}


Fig 1
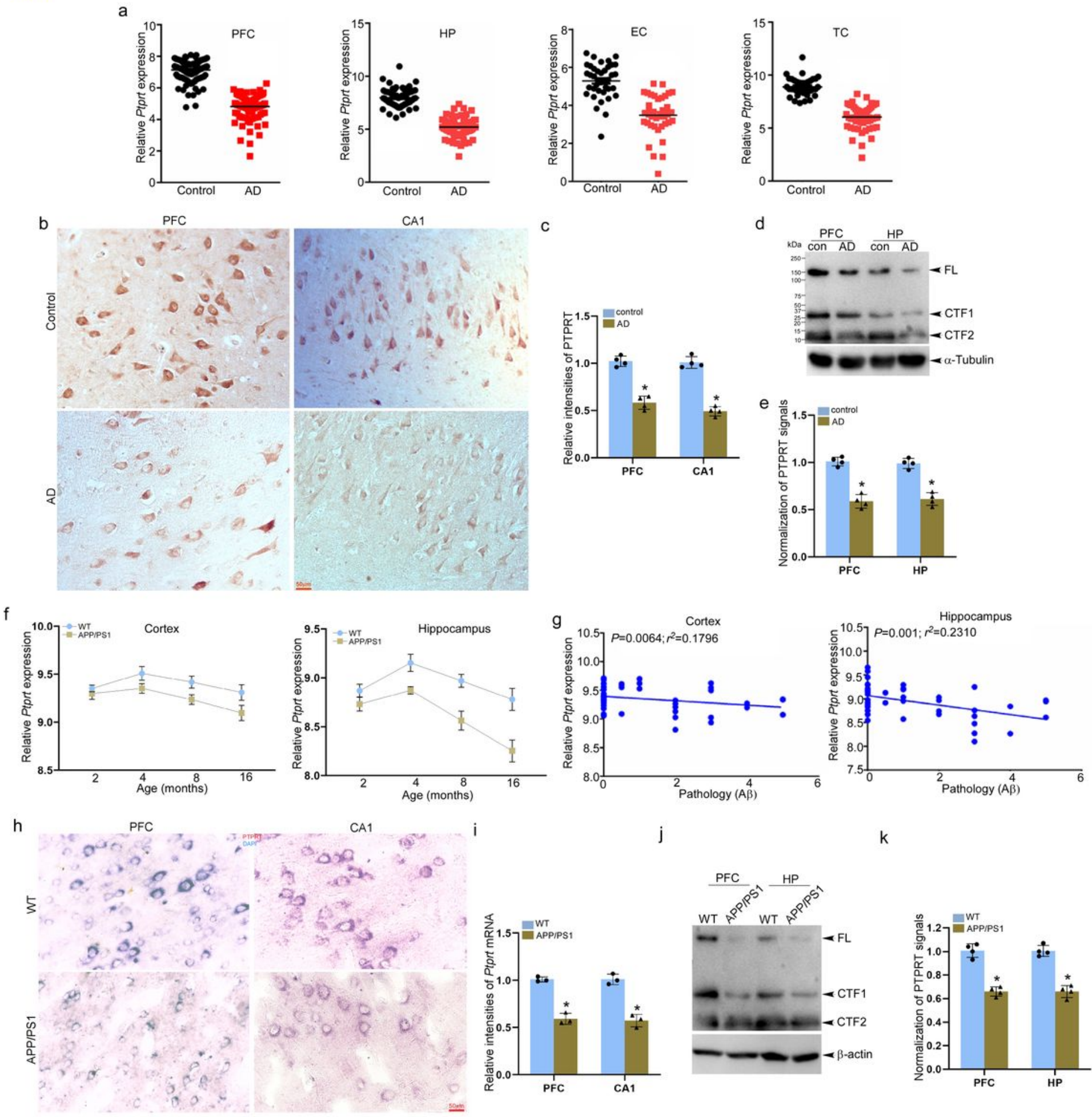

Figure 1

mRNA and protein levels of PTPRT decreased in the brains of human AD and model mice a. The bioinformatic analysis shows the downregulation of Ptprt expression in the brains of AD patients. b. 20 Im paraffin sections of PFC and hippocampus of control and AD patients were immunostained with PTPRT (Brown) antibody. Scale bar, 50ロm. c. Quantification of normalized intensities of PTPRT illustrated in b). Error bars denote S.E.M. ( $n=4$ patients per group; $\left.{ }^{*} P<0.001\right)$. d. Protein extracts from frozen 
postmortem PFC and hippocampal tissues of control and AD patients were assayed by Western blot for the presence of PTPRT and its fragments. e. Quantification of the immunoblot intensities of PTPRT shown in panel d). Error bars denote S.E.M. ( $n=4$ patients per group; $\left.{ }^{*}, P<0.001\right)$. $f-g$. The bioinformatic analysis shows the downregulation of Ptprt expression in the brains of APP/PS mice. h. $10 \square \mathrm{m}$ cryostat brain sections from 12-month-old wild type and APP/PS mice were immunostained with PTPRT (Brown) antibody. Scale bar, 50 $\mathrm{m}$. i. Quantification of normalized intensities of PTPRT illustrated in $h$ ). Error bars denote S.E.M. ( $n=3$ mice per genotype; $\left.{ }^{*} P<0.001\right)$. j. Protein extracts from fresh PFC and hippocampal tissues of 12-month-old wild type and APP/PS mice were assayed by Western blot for the presence of PTPRT and its fragments. k. Quantification of the immunoblot intensities of PTPRT shown in panel j). Error bars denote S.E.M. ( $n=3$ mice per genotype; $\left.{ }^{*}, P<0.001\right)$. 
Fig 2

a

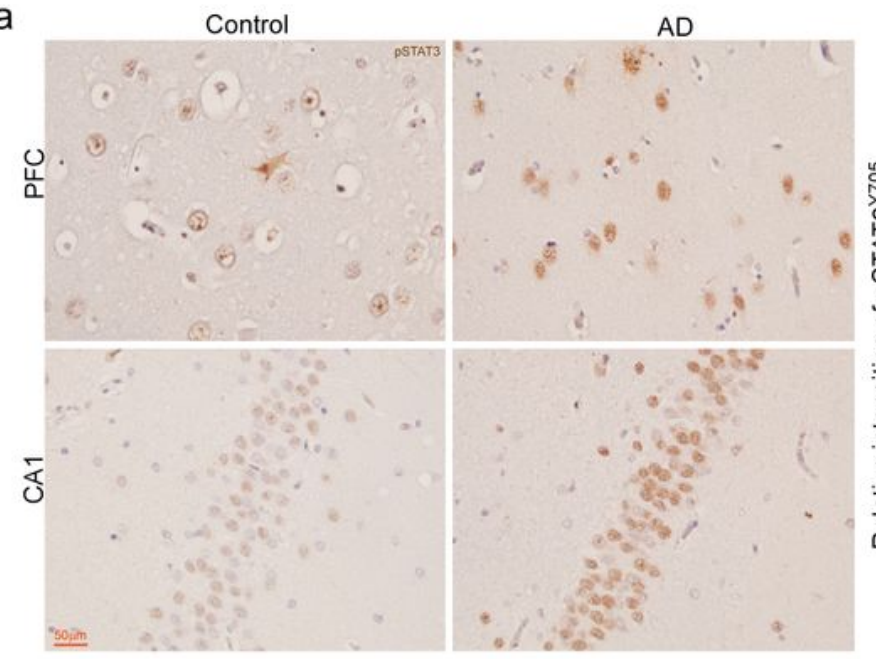

b

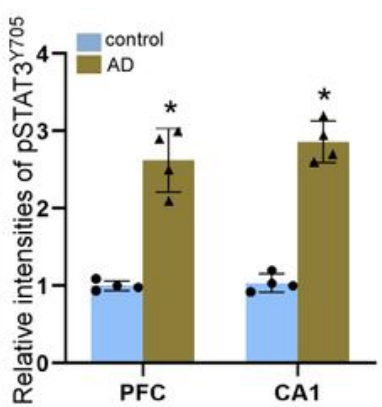

e

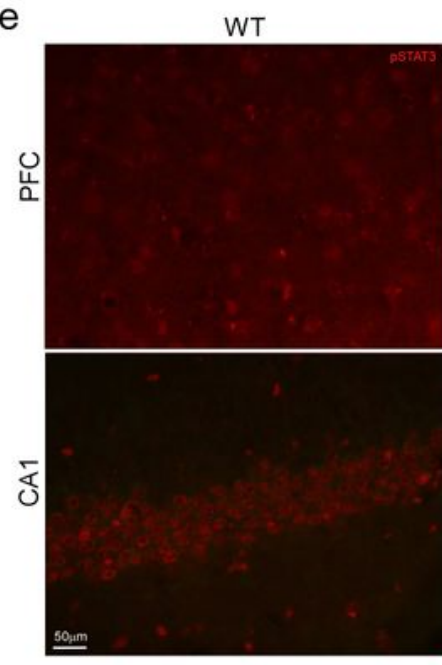

i

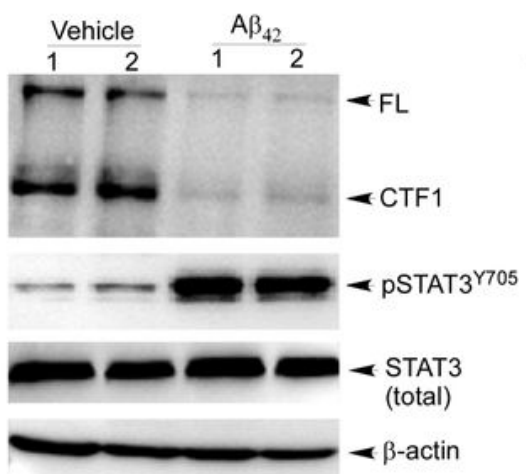

APP/PS1
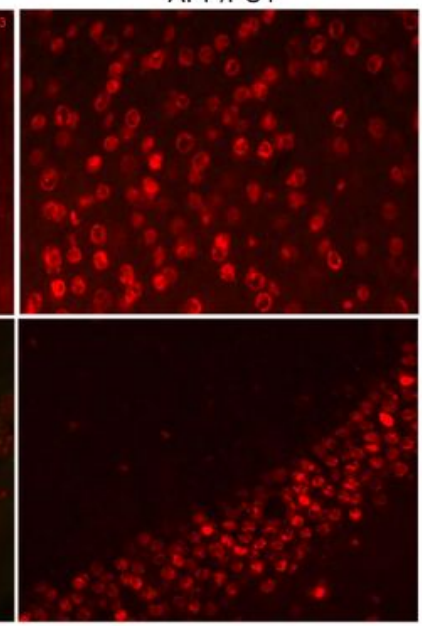

j

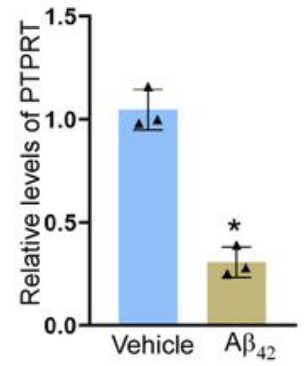

c

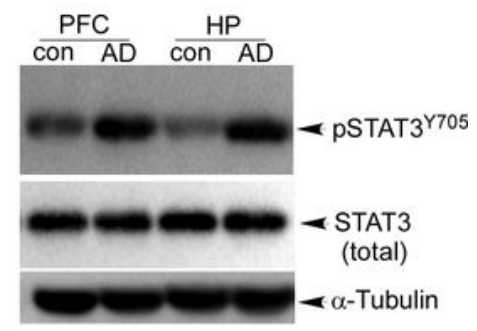

d

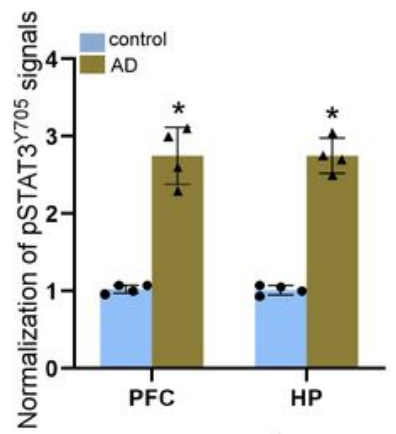

g
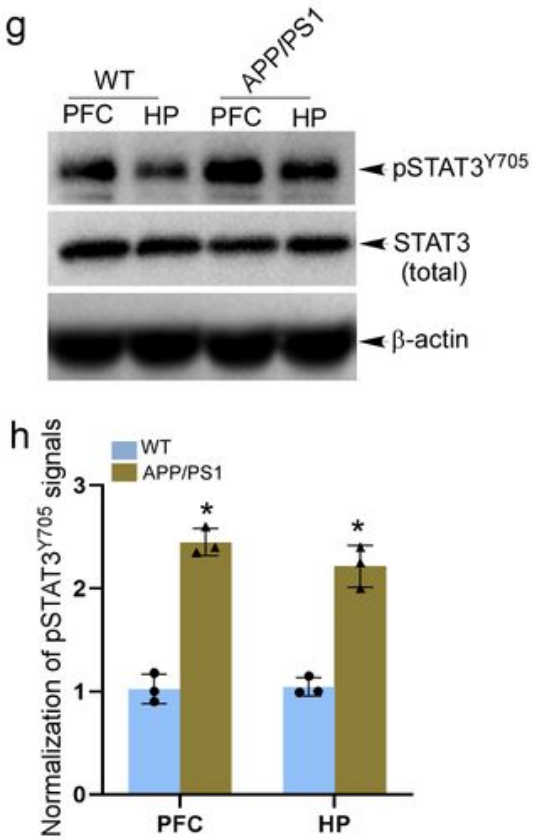

\section{Figure 2}

Increased pSTAT3Y705 found in both human AD and APP/PS mouse brains a. 20 [m paraffin sections of PFC and hippocampus of control and AD patients were immunostained with pSTAT3Y705 (Brown) antibody. Scale bar, 100『m. b. Quantification of normalized intensities of pSTAT3Y705 illustrated in a). Error bars denote S.E.M. ( $n=4$ patients per group; *, $P<0.001)$. c. Protein extracts from frozen postmortem PFC and hippocampal tissues of control and AD patients were assayed by Western blot for 
the presence of pSTAT3Y705. 囚-actin is as the loading control. d. Quantification of the immunoblot intensities of pSTAT3Y705 shown in panel c). Error bars denote S.E.M. ( $n=4$ patients per group; * $P<$ 0.001). e. $10 \square \mathrm{m}$ cryostat brain sections from 12-month-old wild type and APP/PS mice were immunostained with pSTAT3Y705 (Red) antibody. Scale bar, 50ロm. f. Quantification of normalized intensities of pSTAT3Y705 illustrated in e). Error bars denote S.E.M. $(n=3$ mice per genotype; *, $P<0.001)$. g. Protein extracts from fresh PFC and hippocampal tissues of 12-month-old wild type and APP/PS mice were assayed by Western blot for the presence of pSTAT3Y705. 囚-actin was loading control. $h$.

Quantification of the immunoblot intensities of pSTAT3Y705 shown in panel g). Error bars denote S.E.M. $(n=3$ mice per genotype; *, $P<0.001)$. i. Protein extracts from DIV14 cortical neurons of E16.5 wild type and APP/PS fetuses with ADtreatment $\triangle 10 \mu \mathrm{M}, 24$ hours) were assayed by Western blot for the presence of pSTAT3Y705. 区-actin is as the loading control. j-k. Quantification of the immunoblot intensities of pSTAT3Y705 shown in panel i). Error bars denote S.E.M. $\left(n=3\right.$ mice per genotype; $\left.{ }^{*}, P<0.001\right)$.

\section{Fig 3}

a
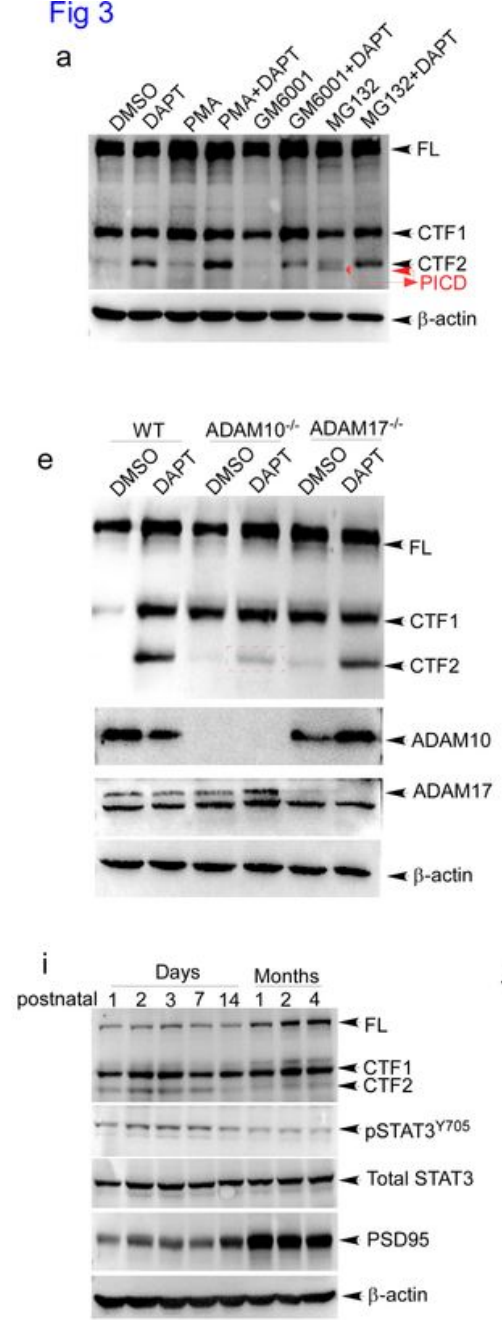

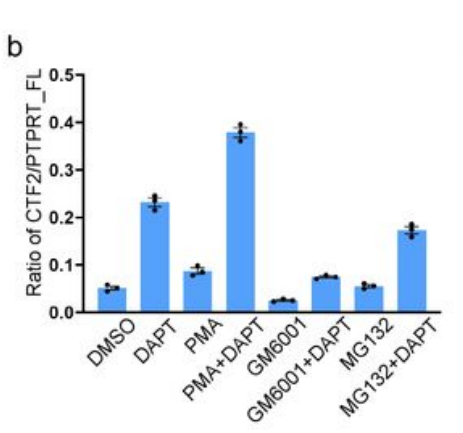

f

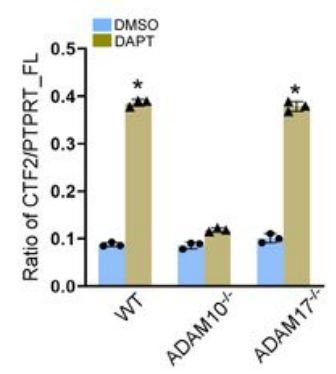

j

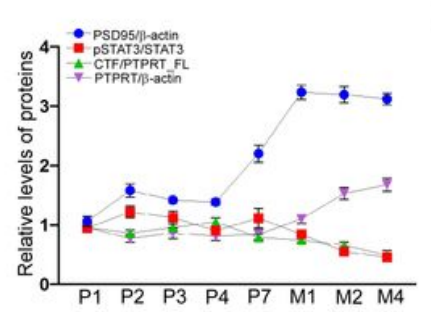

c

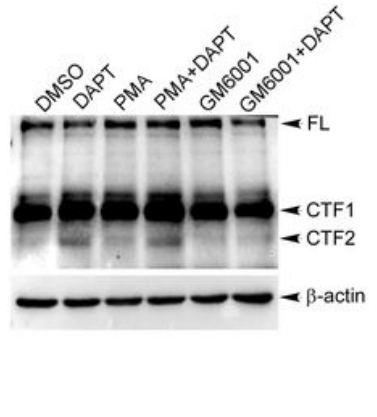

g
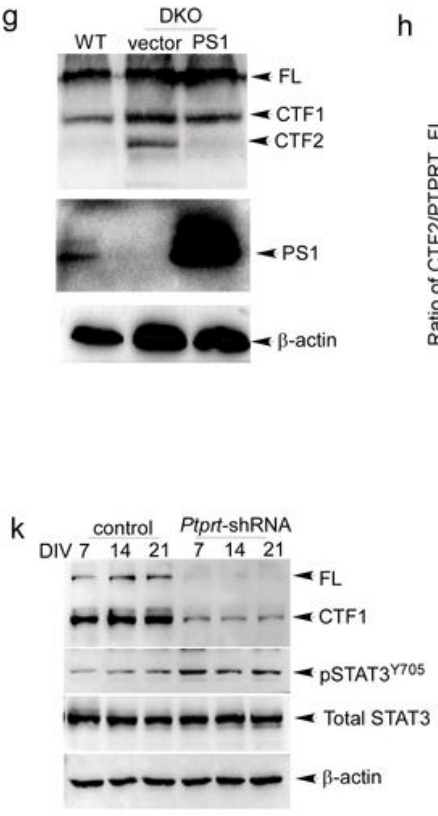

h
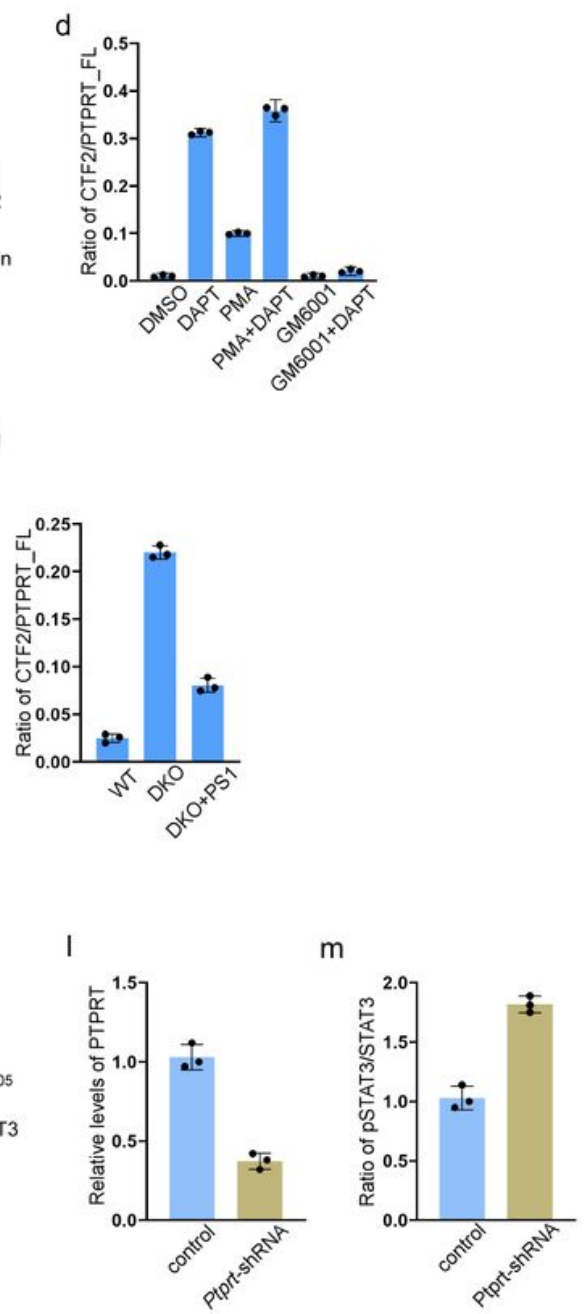

\section{Figure 3}

PTPRT was cleaved by ADAM10 and presenilin 1/ -secretase a. Protein extracts from HEK 293 cells with indicated pretreatments were assayed by Western blot for the presence of PTPRT and its fragments CTF1, CTF2. b. Quantification of the immunoblot intensities of PTPRT shown in panel a). Error bars 
denote S.E.M. ( $n=3$ independent experiments; $\left.{ }^{*} P<0.001\right)$. c. Protein extracts from primary cortical neurons of the wild type at DIV 14 with indicated treatment were assayed by Western blot for the presence of PTPRT and its fragments. $d$. Quantification of the immunoblot intensities of PTPRT shown in panel c). Error bars denote S.E.M. ( $n=3$ independent experiments; $\left.{ }^{*}, P<0.001\right)$. e. Protein extracts from wild type and ADAM10-/- and ADAM17-/- HEK293 cells w/o DAPT treatment were assayed by Western blot for the presence of PTPRT and its fragments. f. Quantification of the immunoblot intensities of PTPRT shown in panel e). Error bars denote S.E.M. $\left(n=3\right.$ independent experiments; $\left.{ }^{*}, P<0.001\right)$. g. Protein extracts from presenilin 1 and 2 DKO MEFs with overexpressing PS1 (presenilin 1) were assayed by Western blot for the presence of PTPRT and its fragments. h. Quantification of the immunoblot intensities of PTPRT shown in panel g). Error bars denote S.E.M. ( $n=3$ independent experiments; ${ }^{*}, P<$ 0.001). i. Protein extracts fresh hippocampal tissues of wild type mice at indicated ages were assayed by Western blot for the presence of PTPRT and its fragments, and PSTAT3Y705. j. Quantification of the immunoblot intensities of PTPRT and its fragments, pSTAT3Y705 shown in panel i). Error bars denote S.E.M. $\left(n=3\right.$ independent experiments; $\left.{ }^{*}, P<0.001\right)$. k. Protein extracts from primary cortical neurons of wild type with infection of lentiviral shRNAs at indicated DIV were assayed by Western blot for the presence of PTPRT and its fragments, and PSTAT3Y705. I-m. Quantification of the immunoblot intensities of PTPRT and its fragments, pSTAT3Y705 shown in panel $k)$. Error bars denote S.E.M. $(n=3$ independent experiments; $\left.{ }^{*}, \mathrm{P}<0.001\right)$. 
Fig 4

a

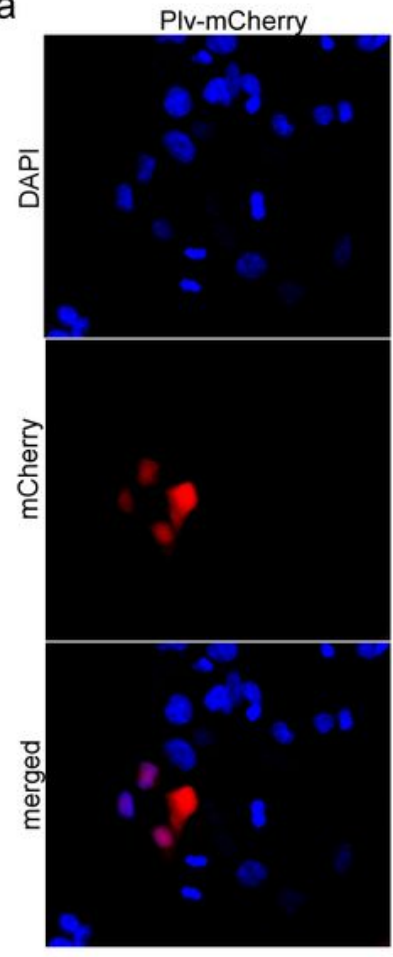

b

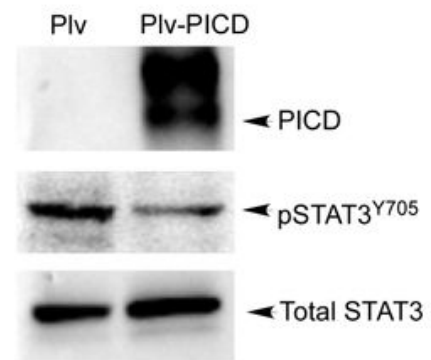

d

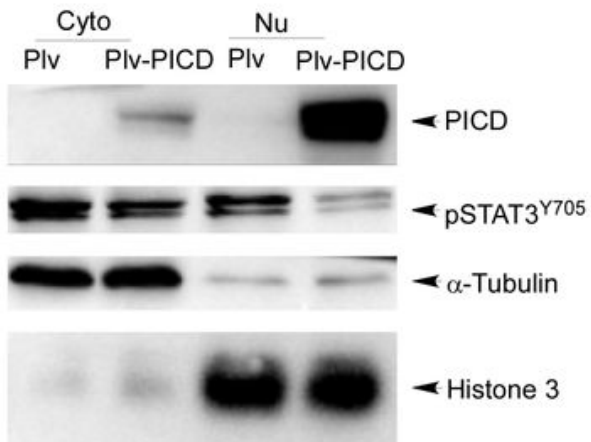

FL-mCherry
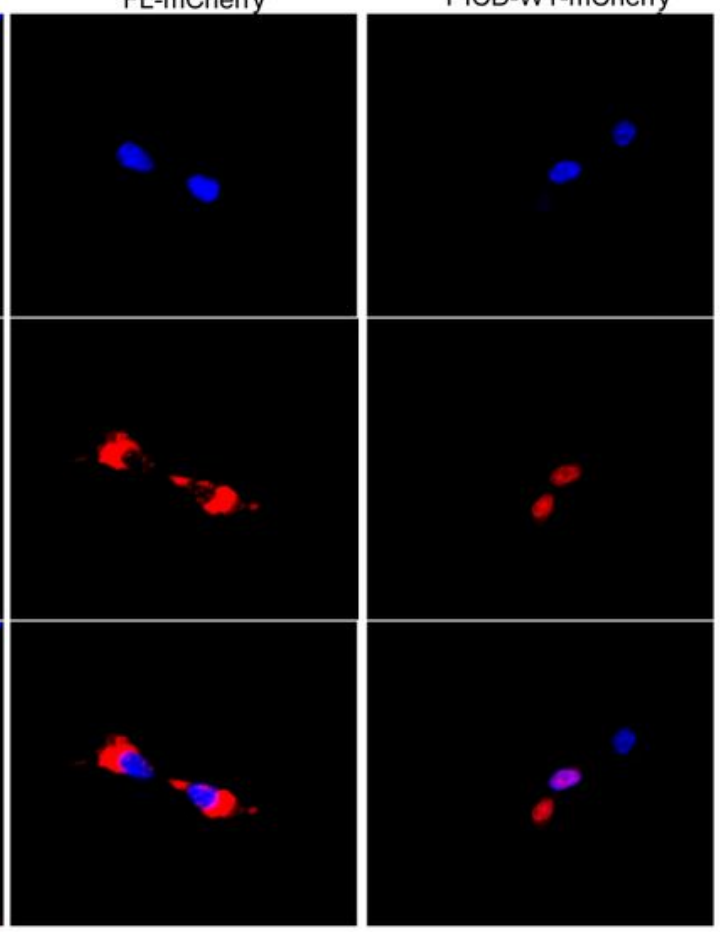

PICD- NLS-mCherry

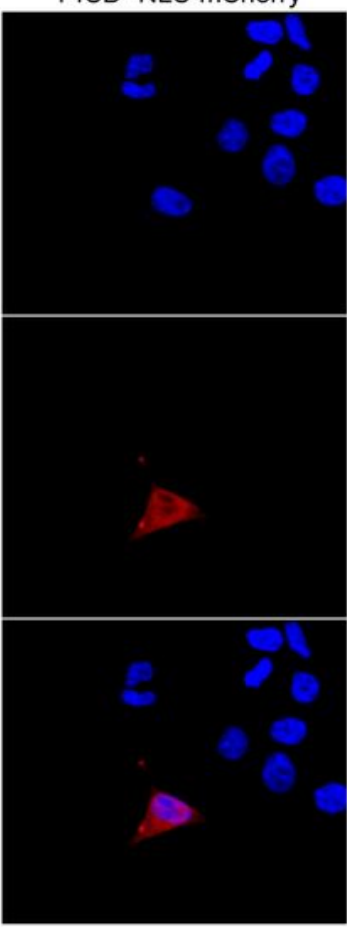

C $\frac{\pi}{2}$

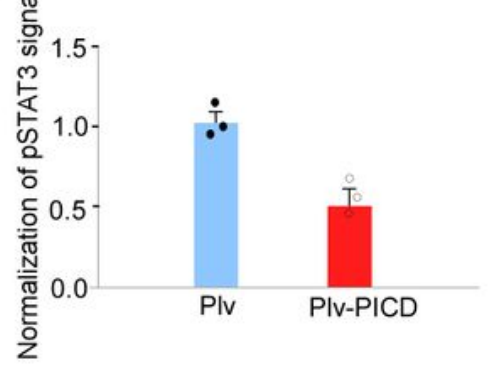

e

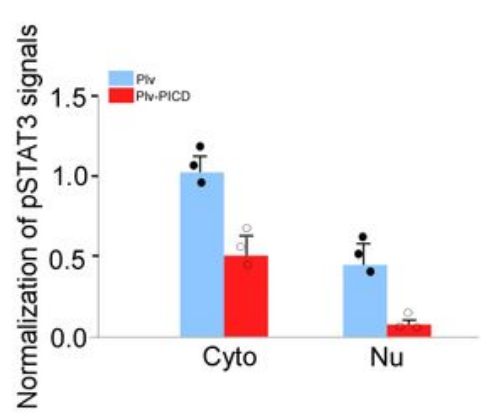

Figure 4

PICD leads to decreases in the levels of pSTAT3 a. PIv-, PTPRT-, PICD-, and PICDNLS-mCherry-expressing N2a cells were fixed and stained with mCherry antibody. b. Protein extracts from Plv- and PICD-mCherry N2a cells were assayed by Western blot for the presence of PICD and pSTAT3Y705. c. Quantification of the immunoblot intensities of pSTAT3Y705 shown in panel b). Error bars denote S.E.M. ( $n=3$ independent experiments; *, P < 0.005). d. Protein extracts from cytoplasmic and nuclear fractions of Plv- and PICD- 
mCherry-expressing N2a cells were assayed by Western blot for the presence of PICD and PSTAT3Y705. e. Quantification of the immunoblot intensities of pSTAT3Y705 shown in panel d). Error bars denote S.E.M. ( $n=3$ independent experiments; $\left.{ }^{*}, \mathrm{P}<0.005\right)$.

Fig 5

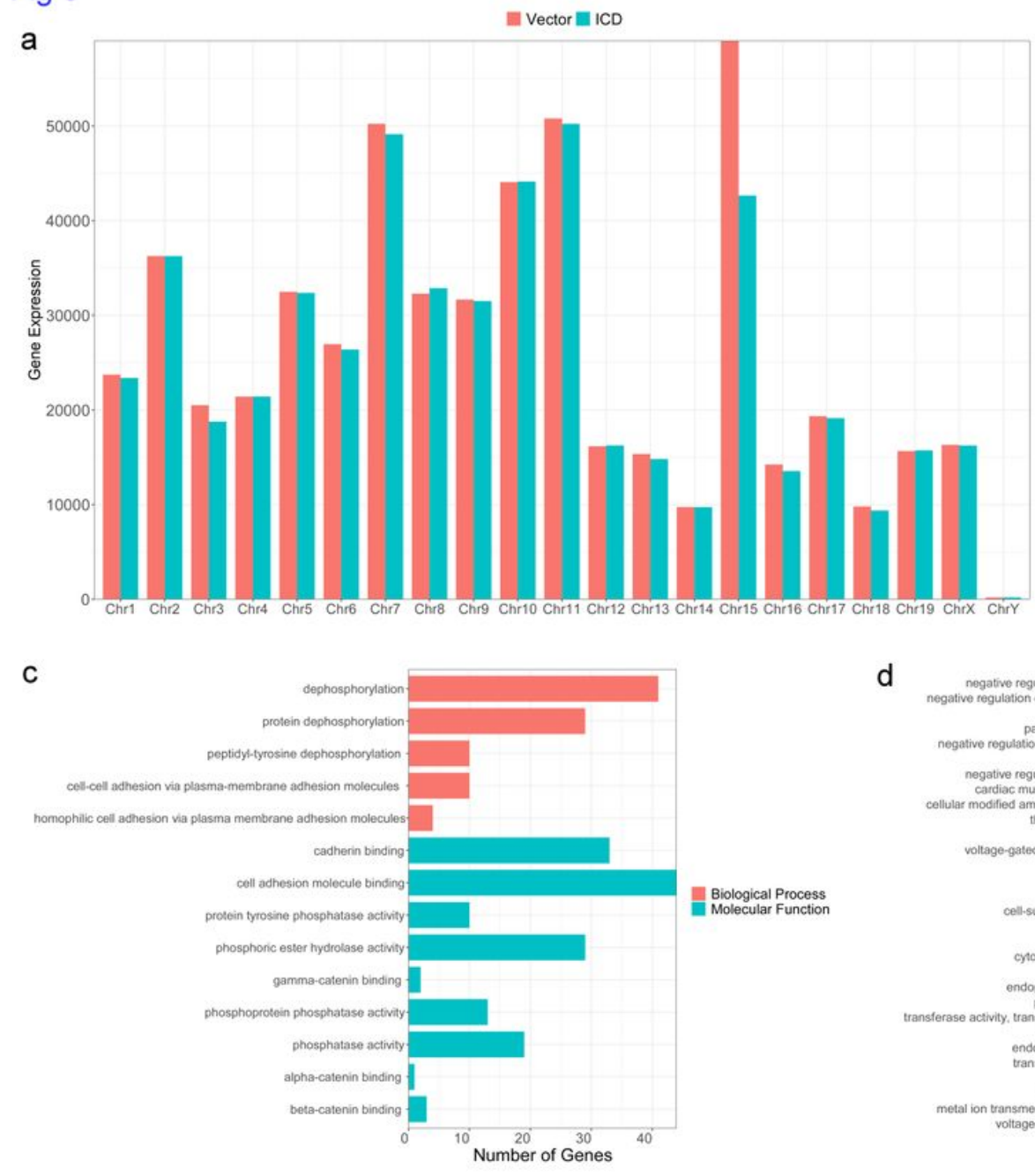

in Vector $\mathrm{in} \mathrm{ICD}$ b

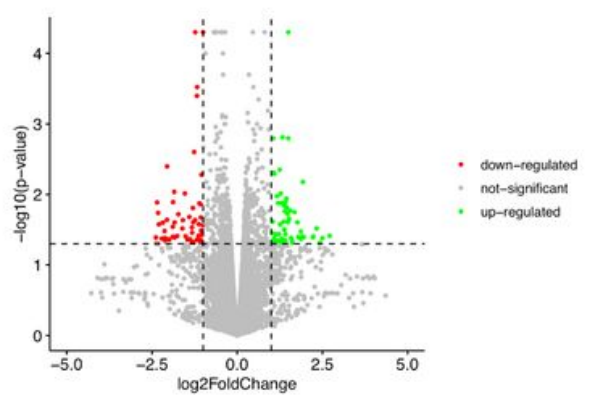

e

Eilogical Process
Molecular Function

d

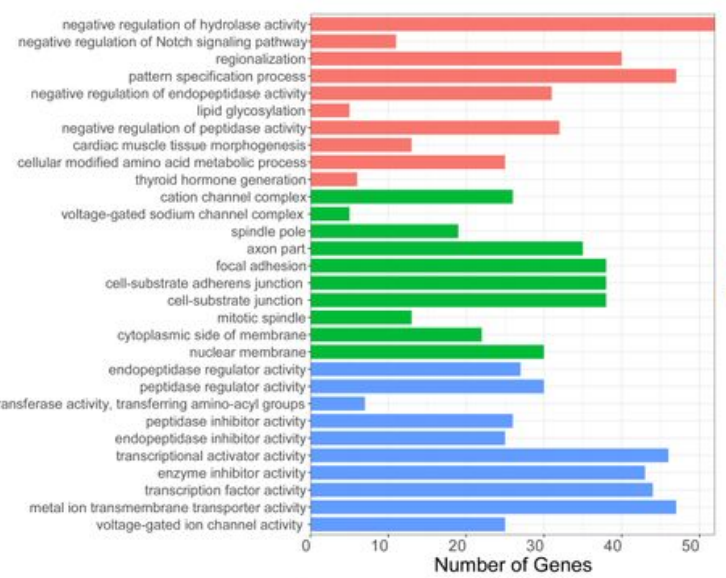

Biological Process
Cellular Componen Cellular Component
Molecular Function

f

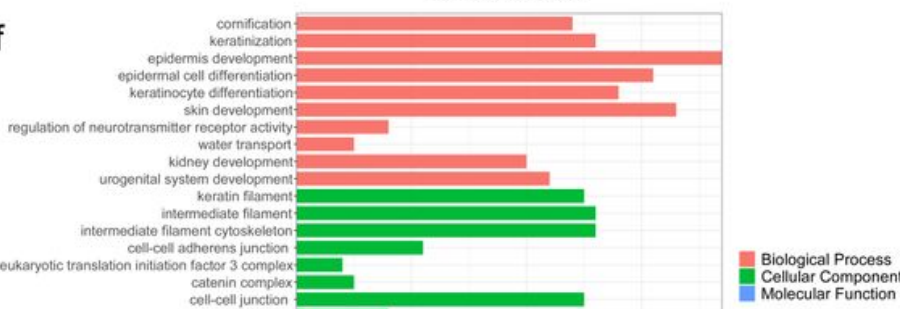

Biological Process

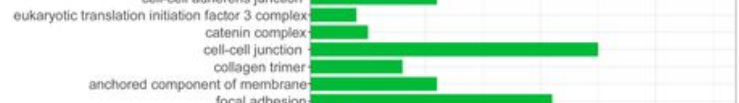

acotycholine receptor roguatator activity

protein C-terminus binding
pocting

solute:-eroton symporter activiny

structural constituent of epidermis

transcriptional activator activity

acetylcholine receptor binding

symporter activity

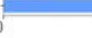

\section{Figure 5}

PICD regulates gene expression in neuronal cells a. The sum of expression of all genes located in each chromosome. b. The genes with significantly different expression between the control vector and PICD groups, red points represent down-regulated genes in the PICD group, green points represent up-regulated 
genes in the PICD group, grey points represent little significant difference. c. The GO annotation contains the Ptprt gene on chromosome 2. d. The number genes annotated to top 10 biological processes, cellular component, and molecular functions, respectively, on chromosome 2. e. The GO annotation contains the Smagp gene on chromosome 15. f. The number genes annotated to top 10 biological processes, cellular component, and molecular functions, respectively, on chromosome 15.

Fig 6

a
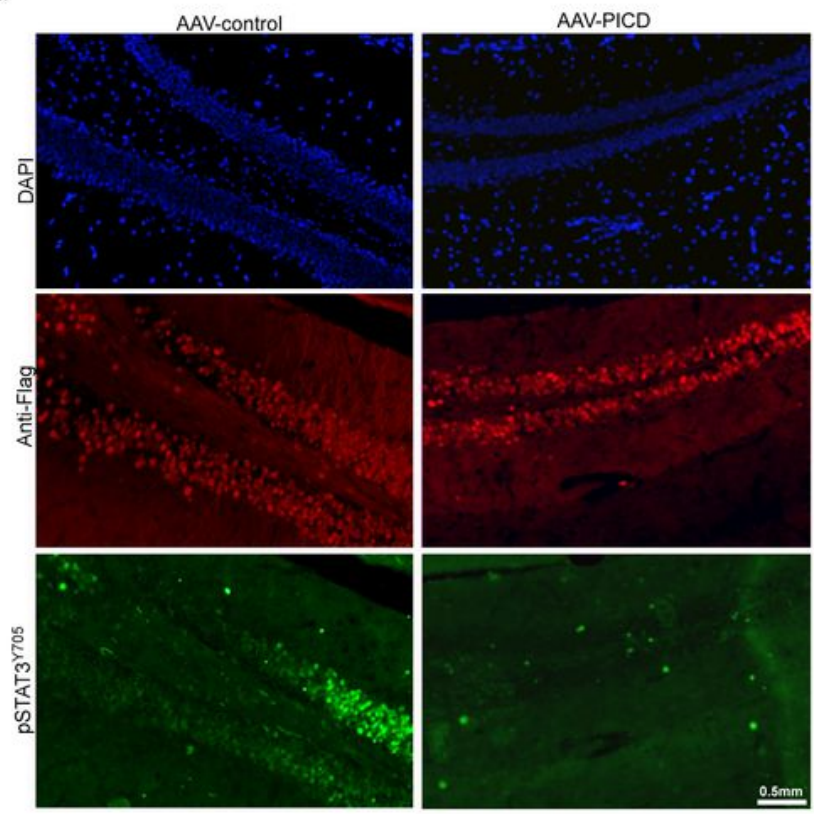

b $\frac{\text { WT }}{\text { AAV-control }} \quad \frac{\text { APP/PS1 }}{\text { AAV-control }} \quad \begin{array}{lll}\text { AAV-PICD } \\ \text { CA1 DG }\end{array}$

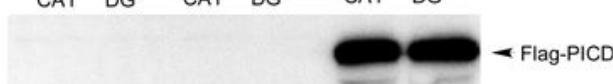

- -
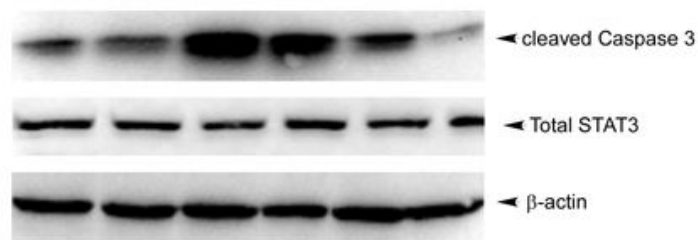

$c$

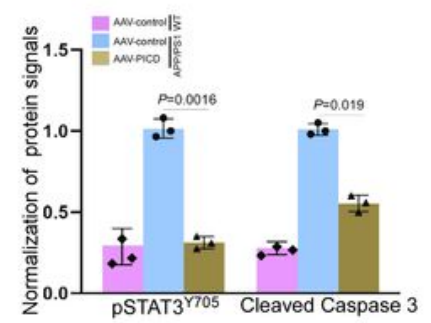

d

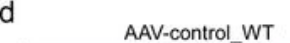

AAV-control_APP/P1

AAV-PICD_APP/PS1

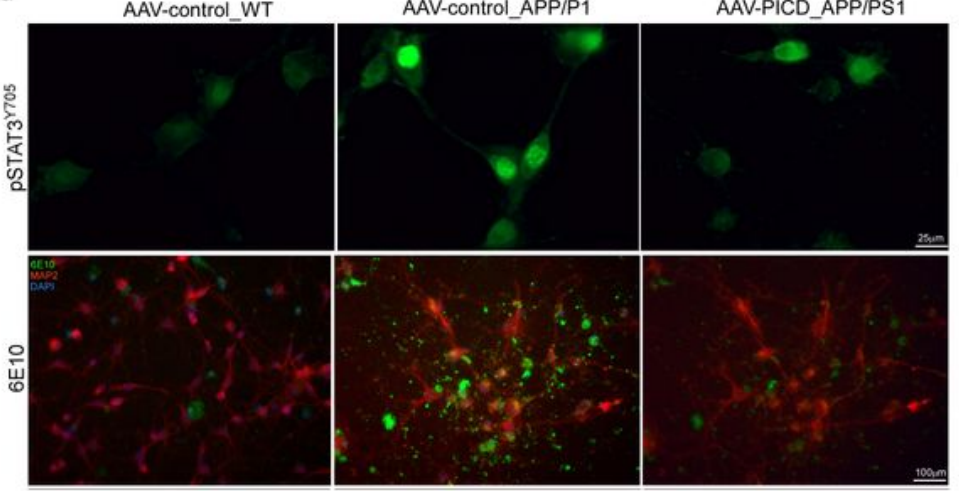

AAV-control_WT

AAV-control_APP/P1

AAV-PICD_APP/PS1

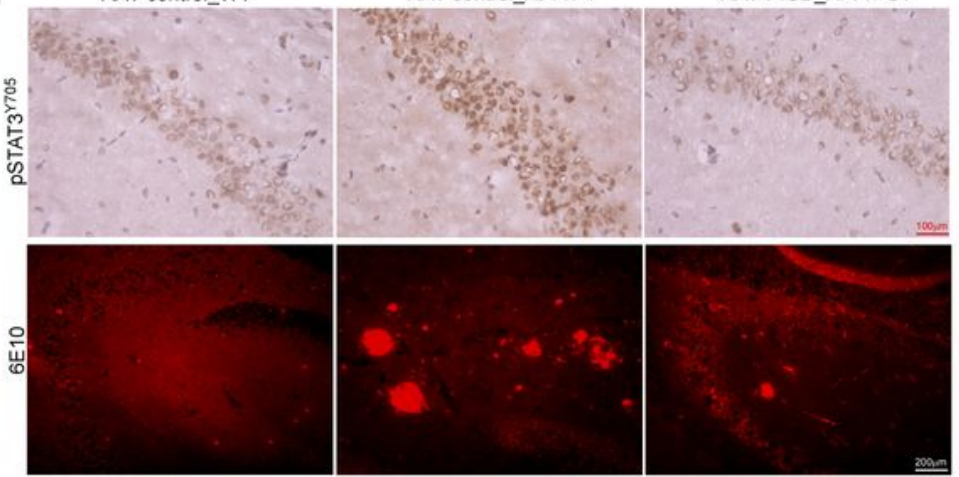

e

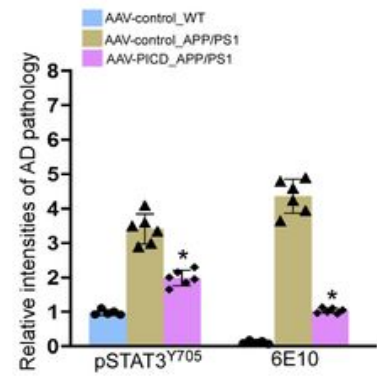

g

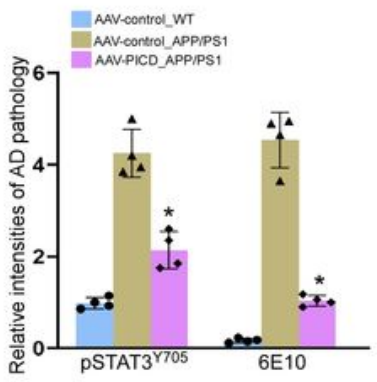

Figure 6 
PICD leads to decreases in the levels of pSTAT3 and A $\beta$ deposition a. The viral particles of AAV-control or AAV-PICD with Flag-tag was injected into the stratum oriens of hippocampus of 9-month-old APP/PS mice, and PICD expression were labelled with Flag antibody and showed the efficiency of gene transfer via immunostained with and Flag-tag (Green) and pSTAT3Y705 (Red) at 12 months. b. Protein extracts from fresh hippocampal CA1 and DG tissues of 12-month-old wild type and microinjection of AAV-control or AAV-PICD were assayed by Western blot for the presence of PICD, pSTAT3Y705, and cleaved Caspase3. 囚-actin was loading control. c. Quantification of the immunobloted intensities of pSTAT3Y705 and cleaved Caspase-3 shown in panel b). Error bars denote S.E.M. $\left(n=3\right.$ mice per genotypes; $\left.{ }^{*}, P<0.001\right)$. $d$. Representative IF images showing the levels of pSTAT3 and 6E10 in cortical neurons of wild type and APP/PS1 mice. Primary cortical neurons from wild type and APP/PS1 at DIV 5 were infected with viral particles of AAV-control, or AAV-PICD and cells were fixed and immunostained with pSTAT3 and 6E10 antibodies at DIV14. Bars were labeled as indicated. e. Quantification of intensities of pSTAT3 and 6E10 immunoreactivities in the hippocampus normalized to an area in square millimeters. Data are presented as mean \pm S.E.M. $\left({ }^{*}, \mathrm{P}<0.005\right)$ compared with viral vector-injected wild type mice as measured by Student's t-test ( $n=6$ independent E16.5 primary cultures per genotype). f. Representative IHC images showing the levels of pSTAT3Y705 and 6E10 in the hippocampus of wild type and APP/PS1 mice. The viral particles of AAV-control or AAV-PICD was injected into the hippocampal regions of 9-month-old mice, and cryostat slices were either immunostained with pSTAT3Y705 and 6E10 antibodies at 12 months.

Bars were labeled as indicated. g. Quantification of intensities of pSTAT3Y705 and 6E10 immunoreactivities in the hippocampus normalized to an area in square millimeters. *, $P<0.001$ compared with viral vector-injected wild type mice as measured by Student's t-test. (WT, AAV-control: $n=4$; APP/PS1, AAV-control, $n=4$; APP/PS1, AAV-PTPRT-ICD, $n=4$ ). 
a

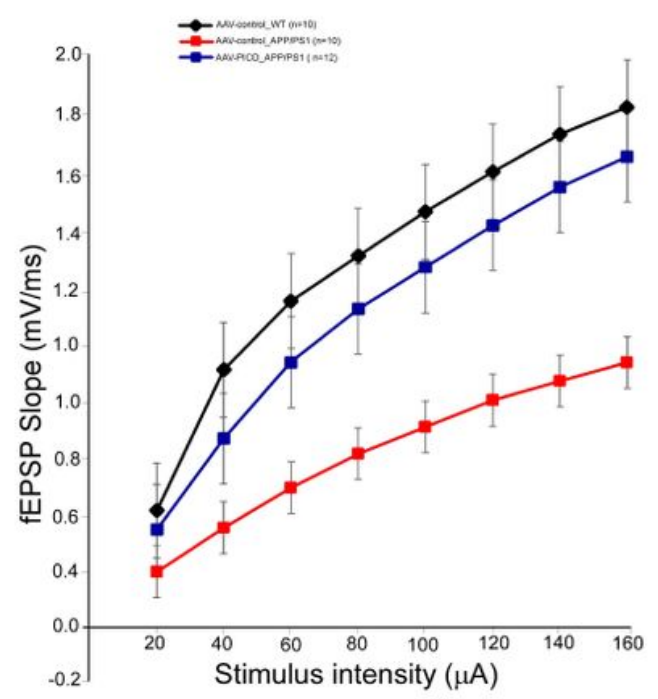

b

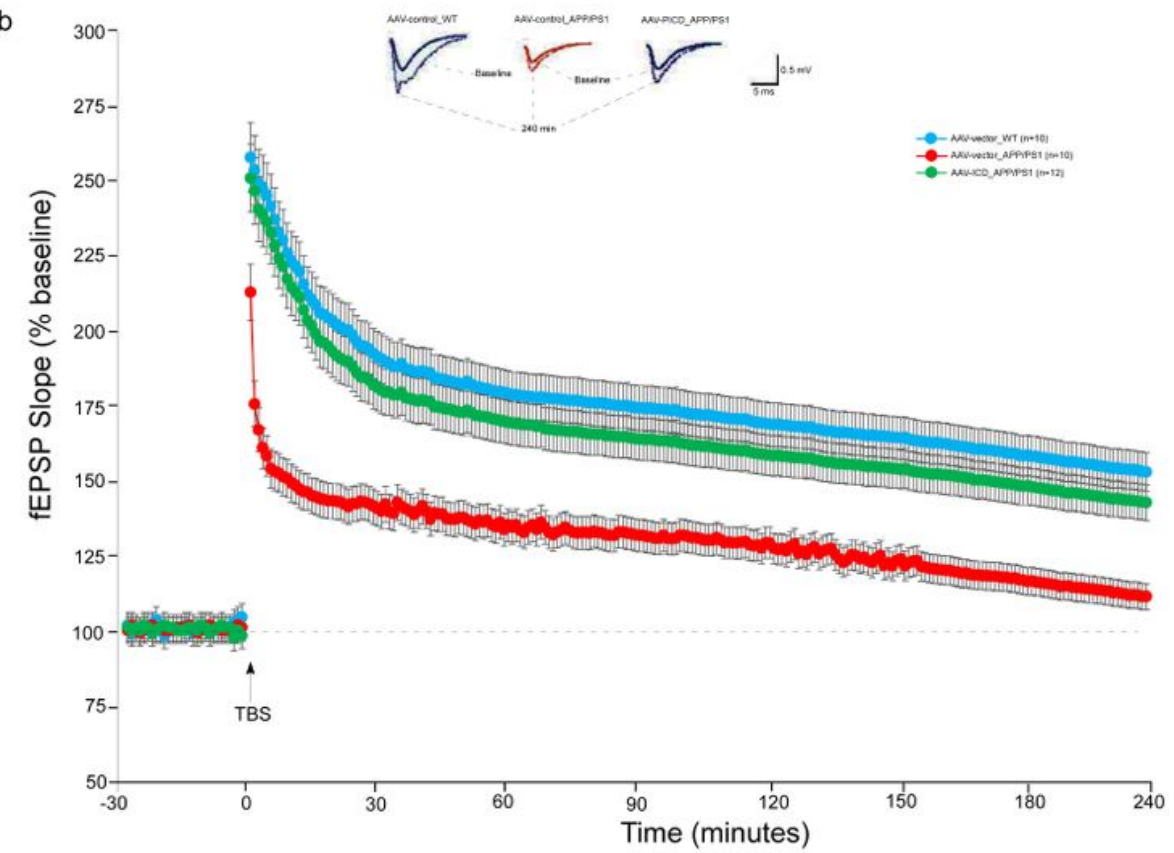

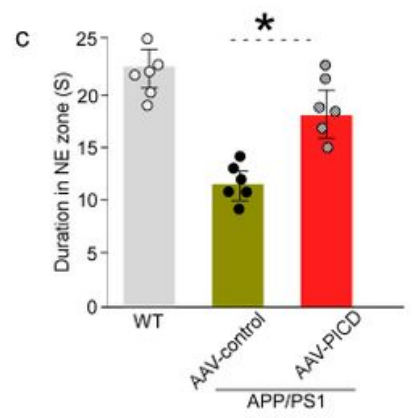

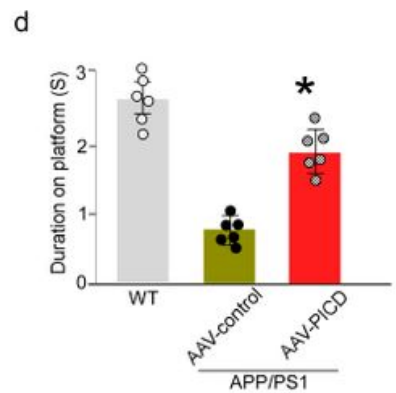

e

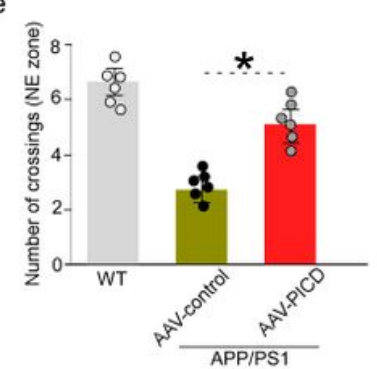

$f$

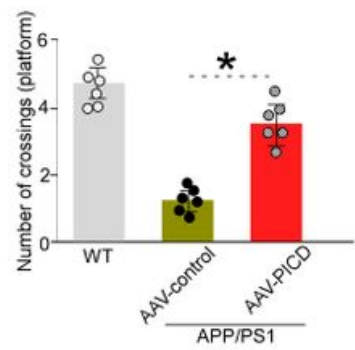

\section{Figure 7}

PICD improves synaptic function and working memory in APP/PS1 mice a. Input/output (I/O) curves and representative fEPSPs at increasing stimulus strengths are shown for WT, APP/PS1 mice with injected viral vector, PICD, respectively. Bigger fEPSPs are evoked in 12-month-old APP/PS1 mice with microinjection of the viral PICD particles compared to the control viral particles, indicating improvement of impaired synaptic transmission. WT, viral control, $1.43 \mathrm{mV} / \mathrm{ms} \pm 0.16 \mathrm{mV} / \mathrm{ms}, \mathrm{n}=8$ slices from 4 mice (10/5); APP/PS1, viral control 0.51mV/ms $\pm 0.08 \mathrm{mV} / \mathrm{ms}, \mathrm{n}=10 / 5, \mathrm{P}<0.001 ;$ APP/PS1, PICD, $1.26 \mathrm{mV} / \mathrm{ms}$ $\pm 0.13 \mathrm{mV} / \mathrm{ms}, \mathrm{n}=12 / 6, \mathrm{P}<0.001$. b. fEPSP slopes were recorded and were expressed as the percentage of the pretetanus baseline. Representative fEPSPs before (solid line) and $60 \mathrm{~min}$ after the induction of LTP (dotted line) are shown. Impaired LTP in 3xTg mice was markedly improved in the overexpression of human PICD catalytic domains. The amount of potentiation of fEPSPs between 0 and 10 min after HFS was $143 \% \pm 15 \%$ in AAV control-expressing APP/PS1 mice $(n=10 / 5)$, and was significantly improved in PICD-expressing APP/PS1 mice $(235 \% \pm 16 \%, n=12 / 6, P<0.001)$, compared to WT mice $(256 \% \pm 19 \%, n$ $=10 / 5, \mathrm{P}=0.0058$ ). The amount of potentiation between 50 to $60 \mathrm{~min}$ after HFS was $229 \% \pm 16 \%$ in PICD-expressing APP/PS1 mice and was not significantly different from WT mice $(235 \% \pm 18 \%, \mathrm{P}=$ 0.167), compared to AAV control expressing APP/PS1 mice (129\% $\pm 10 \%)$. Significance was determined 
using a two-way ANOVA. c-f. PICD alone leads to improvement of spatial learning and memory in APP/PS1 mice. (c-d) Duration in NE zone and the submerged platform area in the probe test. Data were expressed as mean \pm SEM ( $n=6 /$ group). ${ }^{\star} p<0.05$, compared with control. (e-f) Number of crossing the NE zone and submerged platform area times in the probe test. Data were expressed as mean \pm SEM $(n=$ $6 /$ group). ${ }^{*} \mathrm{p}<0.01$, compared with control.

Fig 8

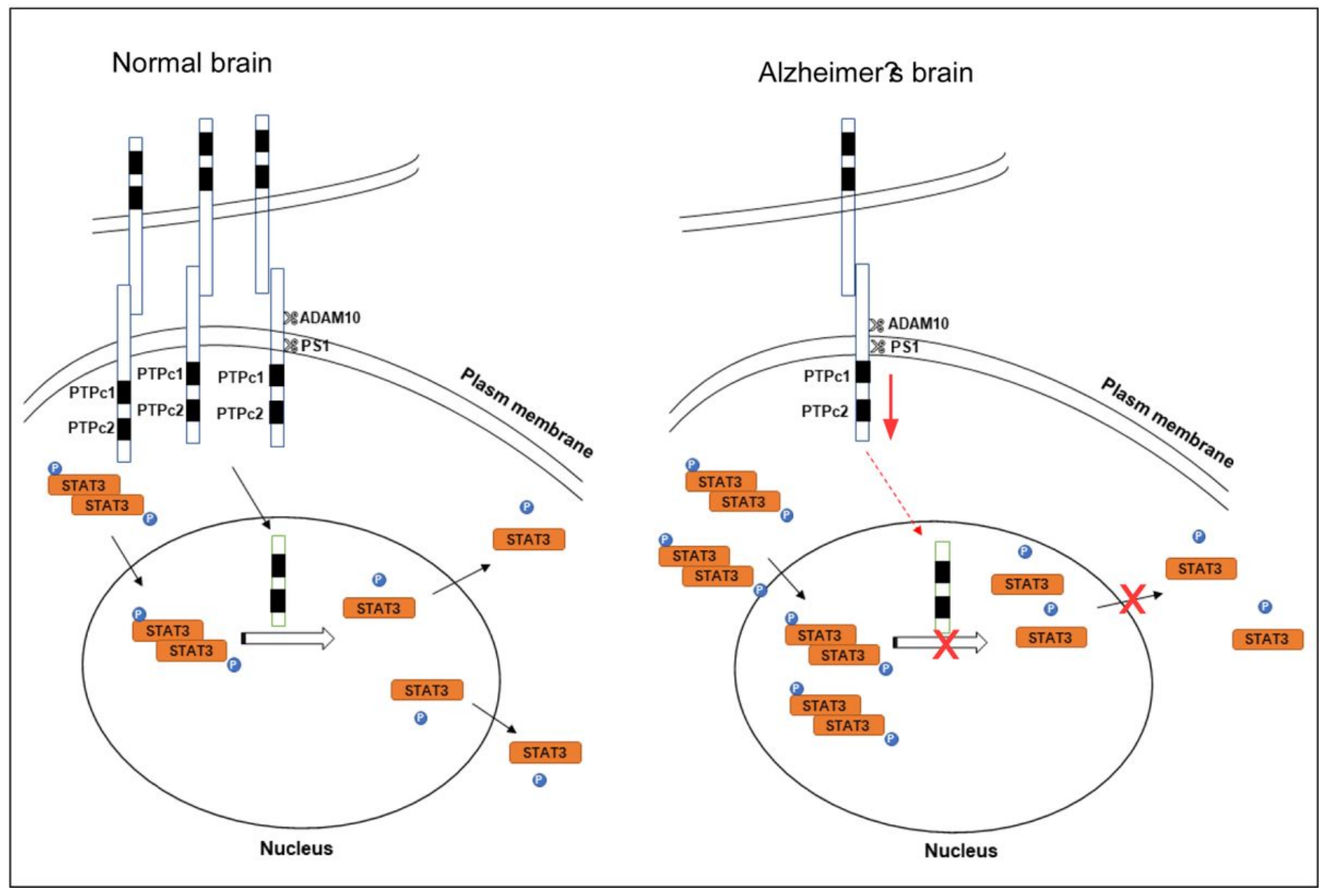

Figure 8

The schematic of PTPRT signaling involved in Alzheimer's pathogenesis In normal brain, PICD released from ADAM10- and presenilin 1-mediated cleavage of PTPRT is translocated to the nucleus of neurons and regulates gene expression via dephosphorylation of pSTAT3Y705. In Alzheimer's brain, loss of PICD resulted from the downregulated levels of PTPRT leads to the accumulation of pSTAT3Y705 in the nucleus.

\section{Supplementary Files}

This is a list of supplementary files associated with this preprint. Click to download. 
- CoverletterMN.pdf

- Supplementarylnformation.pdf 